\title{
ON THE DETERMINATION OF THE SINGER TRANSFER
}

\author{
NGUYẼ̂̃N SUM
}

\begin{abstract}
Let $P_{k}$ be the graded polynomial algebra $\mathbb{F}_{2}\left[x_{1}, x_{2}, \ldots, x_{k}\right]$ with the degree of each generator $x_{i}$ being 1 , where $\mathbb{F}_{2}$ denote the prime field of two elements, and let $G L_{k}$ be the general linear group over $\mathbb{F}_{2}$ which acts regularly on $P_{k}$.

We study the algebraic transfer constructed by Singer [18] using the technique of the Peterson hit problem. This transfer is a homomorphism from the homology of the mod-2 Steenrod algebra $\mathcal{A}$, $\operatorname{Tor}_{k, k+d}^{\mathcal{A}}\left(\mathbb{F}_{2}, \mathbb{F}_{2}\right)$, to the subspace of $\mathbb{F}_{2} \otimes_{\mathcal{A}} P_{k}$ consisting of all the $G L_{k}$-invariant classes of degree $d$.

In this paper, by using the results on the Peterson hit problem we present the proof of the fact that the Singer algebraic transfer is an isomorphism for $k \leqslant 3$. This result has been proved by Singer in 18 for $k \leqslant 2$ and by Boardman in [3] for $k=3$. We show that the fourth Singer transfer is also an isomorphism in certain internal degrees. This result is new and it is different from the ones of Bruner, Hà and Hưng [5], Chơn and Hà [8], Hà [9], Hưng and Quỳnh [12, Nam [16.
\end{abstract}

\section{INTRODUCTION}

Denote by $P_{k}:=\mathbb{F}_{2}\left[x_{1}, x_{2}, \ldots, x_{k}\right]$ the polynomial algebra over the field of two elements, $\mathbb{F}_{2}$, in $k$ generators $x_{1}, x_{2}, \ldots, x_{k}$, each of degree 1 . This algebra arises as the cohomology with coefficients in $\mathbb{F}_{2}$ of an elementary abelian 2-group of rank $k$. Therefore, $P_{k}$ is a module over the mod-2 Steenrod algebra, $\mathcal{A}$. The action of $\mathcal{A}$ on $P_{n}$ is determined by the elementary properties of the Steenrod squares $S q^{i}$ and subject to the Cartan formula $S q^{k}(f g)=\sum_{i=0}^{k} S q^{i}(f) S q^{k-i}(g)$, for $f, g \in P_{k}$ (see Steenrod and Epstein [19).

The Peterson hit problem is to find a minimal generating set for $P_{k}$ regarded as a module over the mod-2 Steenrod algebra. Equivalently, this problem is to find a vector space basis for $Q P_{k}:=\mathbb{F}_{2} \otimes_{\mathcal{A}} P_{k}$ in each degree $d$. Such a basis may be represented by a list of monomials of degree $d$. It is completely determined for $k \leqslant 4$, unknown in general.

Let $G L_{k}$ be the general linear group over the field $\mathbb{F}_{2}$. This group acts naturally on $P_{k}$ by matrix substitution. Since the two actions of $\mathcal{A}$ and $G L_{k}$ upon $P_{k}$ commute with each other, there is an inherited action of $G L_{k}$ on $Q P_{k}$.

Denote by $\left(P_{k}\right)_{d}$ the subspace of $P_{k}$ consisting of all the homogeneous polynomials of degree $d$ in $P_{k}$ and by $\left(Q P_{k}\right)_{d}$ the subspace of $Q P_{k}$ consisting of all the classes represented by the elements in $\left(P_{k}\right)_{d}$. In [18, Singer defined the algebraic

2010 Mathematics Subject Classification. Primary 55T15; Secondary 55S10, 55S05.

Key words and phrases. Steenrod algebra, algebraic transfer, polynomial algebra.

This research is funded by Vietnam National Foundation for Science and Technology Development (NAFOSTED) under grant number 101.04-2017.05. 
transfer, which is a homomorphism

$$
\varphi_{k}: \operatorname{Tor}_{k, k+d}^{\mathcal{A}}\left(\mathbb{F}_{2}, \mathbb{F}_{2}\right) \longrightarrow\left(Q P_{k}\right)_{d}^{G L_{k}}
$$

from the homology of the Steenrod algebra to the subspace of $\left(Q P_{k}\right)_{d}$ consisting of all the $G L_{k}$-invariant classes. It is a useful tool in describing the homology groups of the Steenrod algebra, $\operatorname{Tor}_{k, k+d}^{\mathcal{A}}\left(\mathbb{F}_{2}, \mathbb{F}_{2}\right)$. This transfer was studied by Boardman [3], Bruner, Hà and Hưng [5], Hà [9], Hưng [1], Chơn and Hà [6, 7, 8], Minami [15, Nam [16, Hưng and Quỳnh [12, the present author 22] and others.

Singer showed in 18 that $\varphi_{k}$ is an isomorphism for $k=1,2$. Boardman showed in [3] that $\varphi_{3}$ is also an isomorphism. However, for any $k \geqslant 4, \varphi_{k}$ is not a monomorphism in infinitely many degrees (see Singer [18, Bruner, Hà and Hưng [5], Hưng [11.) Singer made a conjecture in [18] that the algebraic transfer $\varphi_{k}$ is an epimorphism for any $k \geqslant 0$. This conjecture is true for $k \leqslant 3$. It can be verified for $k=4$ by using the results in [21, 23. The conjecture for $k \geqslant 5$ is an open problem.

In this paper, by using the results on the Peterson hit problem we present the proof of the fact that the Singer algebraic transfer is an isomorphism for $k \leqslant 3$. Recall that this result has been proved by Singer in [18] for $k \leqslant 2$ and by Boardman in [3] for $k=3$. To prove this result, Boardman [3] computed the space $Q P_{3}^{G L_{3}}$ by using a basis consisting of the all the classes represented by certain polynomials in $P_{3}$. We also compute this space, however we use the admissible monomial basis for $Q P_{3}$ that is different from the one of Boardman in 3. By applying this technique for $k=4$, we show that the fourth Singer transfer is also an isomorphism in certain internal degrees. This result is new and it is different from the ones of Bruner, Hà and Hưng [5, Chơn and Hà 8], Hà [9], Hưng and Quỳnh [12, Nam 16. In those works it is shown only that the fourth Singer transfer detects certain families of elements in $\operatorname{Ext}_{\mathcal{A}}^{4, *}\left(\mathbb{F}_{2}, \mathbb{F}_{2}\right)$, and fails to detect others.

This paper is organized as follows. In Section 2, we recall some needed information on the lambda algebra and the Singer algebraic transfer. In Sections 3 , we present the determination of the algebraic transfer for $k \leqslant 3$. Finally, in Section 4. we show that the fourth Singer transfer is an isomorphism in certain internal degrees.

\section{The Singer Algebraic transfer And The LAmbDA Algebra}

First of all, we briefly recall the definition of the Singer transfer. Let $\widehat{P}_{1}$ be the submodule of $\mathbb{F}_{2}\left[x_{1}, x_{1}^{-1}\right]$ spanned by all powers $x_{1}^{i}$ with $i \geqslant-1$. The usual $\mathcal{A}$-action on $P_{1}=\mathbb{F}_{2}\left[x_{1}\right]$ is canonically extended to an $\mathcal{A}$-action on $\mathbb{F}_{2}\left[x_{1}, x_{1}^{-1}\right]$ (see Singer [18]). $\widehat{P}_{1}$ is an $\mathcal{A}$-submodule of $\mathbb{F}_{2}\left[x_{1}, x_{1}^{-1}\right]$. The inclusion $P_{1} \subset \widehat{P}_{1}$ gives rise to a short exact sequence of $\mathcal{A}$-modules:

$$
0 \longrightarrow P_{1} \longrightarrow \widehat{P}_{1} \longrightarrow \Sigma^{-1} \mathbb{F}_{2} \longrightarrow 0 .
$$

Let $e_{1}$ be the corresponding element in $\operatorname{Ext}_{\mathcal{A}}^{1}\left(\Sigma^{-1} \mathbb{F}_{2}, P_{1}\right)$. By using the cross and Yoneda products, Singer set

$$
e_{k}=\left(e_{1} \times P_{k-1}\right) \circ\left(e_{1} \times P_{k-2}\right) \circ \ldots\left(e_{1} \times P_{1}\right) \circ e_{1} \in \operatorname{Ext}_{\mathcal{A}}^{k}\left(\Sigma^{-k} \mathbb{F}_{2}, P_{k}\right) .
$$

Then, he defined $\hat{\varphi}_{k}: \operatorname{Tor}_{k}^{\mathcal{A}}\left(\mathbb{F}_{2}, \Sigma^{-k} \mathbb{F}_{2}\right) \longrightarrow \operatorname{Tor}_{0}^{\mathcal{A}}\left(\mathbb{F}_{2}, P_{k}\right)=Q P_{k}$ by $\hat{\varphi}_{k}(z)=e_{k} \cap z$. Its image is a submodule of $\left(Q P_{k}\right)^{G L_{k}}$. So, $\hat{\varphi}_{k}$ induces the homomorphism

$$
\varphi_{k}: \operatorname{Tor}_{k}^{\mathcal{A}}\left(\mathbb{F}_{2}, \Sigma^{-k} \mathbb{F}_{2}\right) \longrightarrow Q P_{k}^{G L_{k}} .
$$


Denote by $\left(P_{k}\right)^{*}$ the dual of $P_{k}$ and by $P\left(\left(P_{k}\right)^{*}\right)$ the primitive subspace consisting of all elements in $\left(P_{k}\right)^{*}$ that are annihilated by every positive degree operations in the mod-2 Steenrod algebra. The dual of $\varphi_{k}$ :

$$
T r_{k}:=\left(\varphi_{k}\right)^{*}: \mathbb{F}_{2} \otimes_{G L_{k}} P\left(\left(P_{k}\right)_{d}^{*}\right) \longrightarrow \operatorname{Ext}_{\mathcal{A}}^{k, k+d}\left(\mathbb{F}_{2}, \mathbb{F}_{2}\right)
$$

is also called the $k$-th Singer transfer.

The algebra $\operatorname{Ext}_{\mathcal{A}}^{* * *}\left(\mathbb{F}_{2}, \mathbb{F}_{2}\right)$ is described in terms of the mod-2 lambda algebra $\Lambda$ (see [4). Recall that $\Lambda$ is a bigraded differential algebra over $\mathbb{F}_{2}$ generated by $\lambda_{j} \in \Lambda^{1, j}, j \geqslant 0$, with the relations

$$
\lambda_{j} \lambda_{2 j+1+m}=\sum_{\nu \geqslant 0}\left(\begin{array}{c}
m-\nu-1 \\
\nu
\end{array}\right) \lambda_{j+m-\nu} \lambda_{2 j+1+\nu}
$$

for $m \geqslant 0$ and the differential

$$
\delta\left(\lambda_{i}\right)=\sum_{\nu \geqslant 0}\left(\begin{array}{c}
i-\nu-1 \\
\nu+1
\end{array}\right) \lambda_{k-\nu-1} \lambda_{\nu}
$$

for $i>0, \delta\left(\lambda_{0}\right)=0$ and that $H^{k, d}(\Lambda, \delta)=\operatorname{Ext}_{\mathcal{A}}^{k, k+d}\left(\mathbb{F}_{2}, \mathbb{F}_{2}\right)$.

For example, the elements $\lambda_{2^{i}-1} \in \Lambda^{1,2^{i}-1}, i \geqslant 0$, and $\bar{d}_{0}=\lambda_{6} \lambda_{2} \lambda_{3}^{2}+\lambda_{4}^{2} \lambda_{3}^{2}+$ $\lambda_{2} \lambda_{4} \lambda_{5} \lambda_{3}+\lambda_{1} \lambda_{5} \lambda_{1} \lambda_{7} \in \Lambda^{4,14}$ are the cycles in the lambda algebra $\Lambda$. So, $h_{i}=$ $\left[\lambda_{2^{i}-1}\right]$ and $d_{0}=\left[\bar{d}_{0}\right]$ are the elements in $\operatorname{Ext}_{\mathcal{A}}^{*, *}\left(\mathbb{F}_{2}, \mathbb{F}_{2}\right)$. Note that $h_{i}$ is the Adams element in $\operatorname{Ext}_{\mathcal{A}}^{1,2^{i}}\left(\mathbb{F}_{2}, \mathbb{F}_{2}\right)$.

There is a homomorphism $\widetilde{S q}^{0}: \Lambda \rightarrow \Lambda$ determined by

$$
\widetilde{S q}^{0}\left(\lambda_{j_{1}} \lambda_{j_{2}} \ldots \lambda_{j_{k}}\right)=\lambda_{2 j_{1}+1} \lambda_{2 j_{2}+1} \ldots \lambda_{2 j_{k}+1}, k \geqslant 0 .
$$

This homomorphism respects the relations in (2.1) and commutes the differential in (2.2). Therefore, it induces a homomorphism

$$
S q^{0}: \operatorname{Ext}_{\mathcal{A}}^{k, k+d}\left(\mathbb{F}_{2}, \mathbb{F}_{2}\right)=H^{k, d}(\Lambda) \longrightarrow H^{k, k+2 d}(\Lambda)=\operatorname{Ext}_{\mathcal{A}}^{k, 2 k+2 d}\left(\mathbb{F}_{2}, \mathbb{F}_{2}\right) .
$$

A family $\left\{a_{i}: i \geqslant 0\right\}$ of elements in $\operatorname{Ext}_{\mathcal{A}}^{k, k+*}\left(\mathbb{F}_{2}, \mathbb{F}_{2}\right)$ is called a $S q^{0}$-family if $a_{i}=$ $\left(S q^{0}\right)^{i}\left(a_{0}\right)$ for every $i \geqslant 0$. It is well known that $\operatorname{Ext}_{\mathcal{A}}^{3,3+*}\left(\mathbb{F}_{2}, \mathbb{F}_{2}\right)$ contains the $S q^{0}$ family of indecomposable elements $\left\{c_{i}\right\}$ and $\operatorname{Ext}_{\mathcal{A}}^{4,4+*}\left(\mathbb{F}_{2}, \mathbb{F}_{2}\right)$ contains seven $S q^{0}$ families of indecomposable elements, namely $\left\{d_{i}\right\},\left\{e_{i}\right\},\left\{f_{i}\right\},\left\{g_{i+1}\right\},\left\{p_{i}\right\},\left\{D_{3}(i)\right\}$, and $\left\{p_{i}^{\prime}\right\}$. Note that $\left\{h_{i}\right\}$ is also a $S q^{0}$-family in $\operatorname{Ext}_{\mathcal{A}}^{1,1+*}\left(\mathbb{F}_{2}, \mathbb{F}_{2}\right)$.

The algebra $\left\{\operatorname{Ext}_{\mathcal{A}}^{k, k+*}\left(\mathbb{F}_{2}, \mathbb{F}_{2}\right) \mid k \geqslant 0\right\}$ has been explicitly computed by Adem [2] for $k=1$, by Adams [1] and Wall [24] for $k=2$, by Adams [1] and Wang 25] for $k=3$ and by Lin 14 for $k=4$.

Theorem 2.1 (See [1, 2, 14, 24, 25]).

i) The algebra $\left\{\operatorname{Ext}_{\mathcal{A}}^{k, k+*}\left(\mathbb{F}_{2}, \mathbb{F}_{2}\right) \mid k \geqslant 0\right\}$ for $k \leqslant 3$ is generated by $h_{i}$ and $c_{i}$ for $i \geqslant 0$ and subject only to the relations $h_{i} h_{i+1}=0, h_{i} h_{i+2}^{2}=0$ and $h_{i}^{3}=$ $h_{i-1}^{2} h_{i+1}$. In particular, $\left\{c_{i}: i \geqslant 0\right\}$ is an $\mathbb{F}_{2}$-basis for the indecomposable elements in $\operatorname{Ext}_{\mathcal{A}}^{3,3+*}\left(\mathbb{F}_{2}, \mathbb{F}_{2}\right)$.

ii) The algebra $\left\{\operatorname{Ext}_{\mathcal{A}}^{k, k+*}\left(\mathbb{F}_{2}, \mathbb{F}_{2}\right) \mid k \geqslant 0\right\}$ for $k \leqslant 4$ is generated by $h_{i}, c_{i}, d_{i}, e_{i}$, $f_{i}, g_{i+1}, p_{i}, D_{3}(i)$ and $p_{i}^{\prime}$ for $i \geqslant 0$ and subject to the relations in $\left.i\right)$ together with the relations $h_{i}^{2} h_{i+3}^{2}=0, h_{j} c_{i}=0$ for $j=i-1, i, i+2$ and $i+3$. Furthermore, the set of the elements $d_{i}, e_{i}, f_{i}, g_{i+1}, p_{i}, D_{3}(i)$ and $p_{i}^{\prime}$, for $i \geqslant 0$, is an $\mathbb{F}_{2}$-basis for the indecomposable elements in $\operatorname{Ext}_{\mathcal{A}}^{4,4+*}\left(\mathbb{F}_{2}, \mathbb{F}_{2}\right)$. 
It is well known that the dual of $P_{k}$ is the divided power algebra generated by $a_{1}, a_{2}, \ldots, a_{k}$ :

$$
\left(P_{k}\right)^{*}=\Gamma\left(a_{1}, a_{2}, \ldots, a_{k}\right)
$$

where $a_{j}^{(i)}$ is dual to $x_{j}^{i} \in P_{k}$ with respect to the basis of $P_{k}$ consisting of all monomials in $x_{1}, x_{2}, \ldots, x_{k}$ and $a_{j}=a_{j}^{(1)}$. The graded vector space $\left\{\left(P_{k}\right)^{*} \mid k \geqslant 0\right\}$ is an algebra with a multiplication defined by

$$
\left(a_{1}^{\left(i_{1}\right)} \ldots a_{k}^{\left(i_{k}\right)}\right)\left(a_{1}^{\left(i_{k+1}\right)} \ldots a_{m}^{\left(i_{k+m}\right)}\right)=a_{1}^{\left(i_{1}\right)} \ldots a_{k}^{\left(i_{k}\right)} a_{k+1}^{\left(i_{k+1}\right)} \ldots a_{k+m}^{\left(i_{k+m}\right)} \in\left(P_{k+m}\right)^{*},
$$

for any $a_{1}^{\left(i_{1}\right)} \ldots a_{k}^{\left(i_{k}\right)} \in\left(P_{k}\right)^{*}$ and $a_{1}^{\left(i_{k+1}\right)} \ldots a_{m}^{\left(i_{k+m}\right)} \in\left(P_{m}\right)^{*}$. In [6], Chơn and Hà defined a homomorphism of algebras

$$
\phi=\left\{\phi_{k} \mid k \geqslant 0\right\}:\left\{\left(P_{k}\right)^{*} \mid k \geqslant 0\right\} \longrightarrow\left\{\Lambda^{k, *} \mid k \geqslant 0\right\}=\Lambda,
$$

which induces the Singer transfer. Here, the homomorphism $\phi_{k}:\left(P_{k}\right)^{*} \rightarrow \Lambda^{k, *}$ is defined by the following inductive formula:

$$
\phi_{k}\left(a^{(I, t)}\right)= \begin{cases}\lambda_{t}, & \text { if } k-1=\ell(I)=0, \\ \sum_{i \geqslant t} \phi_{k-1}\left(S q^{i-t} a^{I}\right) \lambda_{i}, & \text { if } k-1=\ell(I)>0,\end{cases}
$$

for any $a^{(I, t)}=a_{1}^{\left(i_{1}\right)} a_{2}^{\left(i_{2}\right)} \ldots a_{k-1}^{\left(i_{k-1}\right)} a_{k}^{(t)} \in\left(P_{k}\right)^{*}$ and $I=\left(i_{1}, i_{2}, \ldots, i_{k-1}\right)$.

Theorem 2.2 (See Chơn and Hà [6]). If $b \in P\left(\left(P_{k}\right)^{*}\right)$, then $\phi_{k}(b)$ is a cycle in the lambda algebra $\Lambda$ and $\operatorname{Tr}_{k}([b])=\left[\phi_{k}(b)\right]$.

Note that this theorem is a dual version of the one in Hưng [10.

We end this section by recalling some results on Kameko's homomorphism and the generators of the general linear group $G L_{k}$.

One of the main tools in the study of the hit problem is Kameko's homomorphism ${\widetilde{S q_{*}}}_{*}^{0}: Q P_{k} \rightarrow Q P_{k}$. This homomorphism is induced by the $\mathbb{F}_{2}$-linear map $\psi: P_{k} \rightarrow$ $P_{k}$, given by

$$
\psi(x)= \begin{cases}y, & \text { if } x=x_{1} x_{2} \ldots x_{k} y^{2} \\ 0, & \text { otherwise }\end{cases}
$$

for any monomial $x \in P_{k}$. Note that $\psi$ is not an $\mathcal{A}$-homomorphism. However, $\psi S q^{2 t}=S q^{t} \psi$, and $\psi S q^{2 t+1}=0$ for any non-negative integer $t$.

For a positive integer $n$, by $\mu(n)$ one means the smallest number $r$ for which it is possible to write $n=\sum_{1 \leqslant i \leqslant r}\left(2^{u_{i}}-1\right)$, where $u_{i}>0$.

Theorem 2.3 (Kameko [13]). Let $m$ be a positive integer. If $\mu(2 m+k)=k$, then $\left(\widetilde{S q}_{*}^{0}\right)_{m}:\left(Q P_{k}\right)_{2 m+k} \rightarrow\left(Q P_{k}\right)_{m}$ is an isomorphism of the GL $L_{k}$-modules.

Definition 2.4. Let $f, g$ be two polynomials of the same degree in $P_{k}$. Then, $f \equiv g$ if and only if $f-g \in \mathcal{A}^{+} P_{k}$. If $f \equiv 0$, then $f$ is called hit.

For $1 \leqslant i \leqslant k$, define the $\mathcal{A}$-homomorphism $\rho_{i}: P_{k} \rightarrow P_{k}$, which is determined by $\rho_{i}\left(x_{i}\right)=x_{i+1}, \rho_{i}\left(x_{i+1}\right)=x_{i}, \rho_{i}\left(x_{j}\right)=x_{j}$ for $j \neq i, i+1,1 \leqslant i<k$, and $\rho_{k}\left(x_{1}\right)=x_{1}+x_{2}, \rho_{k}\left(x_{j}\right)=x_{j}$ for $j>1$.

It is easy to see that the general linear group $G L_{k}$ is generated by the matrices associated with $\rho_{i}, 1 \leqslant i \leqslant k$, and the symmetric group $\Sigma_{k}$ is generated by the ones associated with $\rho_{i}, 1 \leqslant i<k$. So, a class $[f]$ represented by a homogeneous polynomial $f \in P_{k}$ is an $G L_{k}$-invariant if and only if $\rho_{i}(f) \equiv f$ for $1 \leqslant i \leqslant k$. It is an $\Sigma_{k}$-invariant if and only if $\rho_{i}(f) \equiv f$ for $1 \leqslant i<k$. 


\section{Determination OF $T r_{k}$ FOR $k \leqslant 3$}

\subsection{Determination of $T r_{k}$ for $k \leqslant 2$.}

In this subsection, we present the proof of the following.

Theorem 3.1.1 (Singer [18]). The algebraic transfer $T r_{k}$ is an isomorphism for $k \leqslant 2$.

It is well-known that

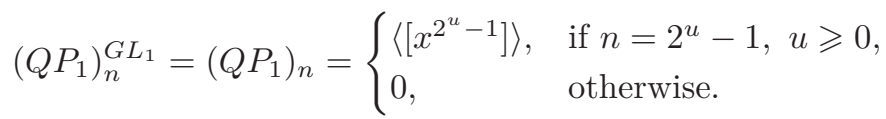

According to Theorem 2.1] we have

$$
\operatorname{Ext}_{\mathcal{A}}^{1, t+1}\left(\mathbb{F}_{2}, \mathbb{F}_{2}\right)= \begin{cases}\left\langle h_{u}\right\rangle, & \text { if } t=2^{u}-1, u \geqslant 0, \\ 0, & \text { otherwise. }\end{cases}
$$

Since $\left(P_{1}\right)^{*}=\Gamma(a)$ and $a^{\left(2^{u}-1\right)} \in P\left(\left(P_{1}\right)^{*}\right), \phi_{1}\left(a^{\left(2^{u}-1\right)}\right)=\lambda_{2^{u}-1}$ is a cycle in $\Lambda^{1, *}$. Using Theorem 2.2 we get

$$
\operatorname{Tr}_{1}\left(\left[a^{\left(2^{u}-1\right)}\right]\right)=\left[\phi_{1}\left(a^{\left(2^{u}-1\right)}\right)\right]=\left[\lambda_{2^{u}-1}\right]=h_{u}, \forall u \geqslant 0 .
$$

So, $T r_{1}$ is a isomorphism.

Now, we present the proof of this theorem for $k=2$ by computing the space $\left(Q P_{2}\right)^{G L_{2}}$. From a result of Wood [26], we need only to compute this space in the degree $n=2^{s+t}+2^{s}-2$ with $s, t$ non-negative integers.

First, we consider the degree $n=2^{s+1}-2$ with $s \geqslant 0$. Since the iterated Kameko homomorphism $\left(\widetilde{S q}_{*}^{0}\right)^{s}:\left(Q P_{2}\right)_{n} \rightarrow\left(Q P_{2}\right)_{0}$ is a isomorphism of $G L_{2}$-modules and $\left(Q P_{2}\right)_{0}^{G L_{2}}=\langle 1\rangle$, hence $\left(Q P_{2}\right)_{n}^{G L_{2}}=\left\langle\left[p_{2, s}\right]\right\rangle$ with $p_{2, s}:=\left(x_{1} x_{2}\right)^{2^{s}-1}$.

Next, we compute $\left(Q P_{2}\right)_{n}^{G L_{2}}$ with $n=2^{s+1}+2^{s}-2, s \geqslant 0$. Since the iterated Kameko homomorphism $\left(\widetilde{S q}_{*}^{0}\right)^{s}:\left(Q P_{2}\right)_{n} \rightarrow\left(Q P_{2}\right)_{1}$ is a isomorphism of $G L_{2^{-}}$ modules, we need only to compute $\left(Q P_{2}\right)_{1}^{G L_{2}}$.

According to Peterson [17, $\left(Q P_{2}\right)_{n}$ is the vector space of dimension 2 with a basis consisting of 2 classes represented by the following monomials:

$$
v_{s, 1}=x_{1}^{2^{s}-1} x_{2}^{2^{s+1}-1}, v_{s, 2}=x_{1}^{2^{s+1}-1} x_{2}^{2^{s}-1} .
$$

In particular, $v_{0,1}=x_{2} . v_{0,2}=x_{1}$. Suppose $\theta=a_{1} v_{1}+a_{2} v_{2}=a_{1} x_{2}+a_{2} x_{1} \in$ $\left(Q P_{2}\right)_{1}^{G L_{2}}$ with $a_{1}, a_{2} \in \mathbb{F}_{2}$. Then $\rho_{1}(\theta)=a_{1} v_{2}+a_{2} v_{1} \equiv \theta$. So, we get $a_{1}=a_{2}$. Since $\rho_{2}(\theta) \equiv a_{1} v_{1}+a_{2}\left(v_{1}+v_{2}\right) \equiv \theta$, we obtain $a_{1}=a_{2}=0$. Hence, $\left(Q P_{2}\right)_{1}^{G L_{2}}=0$ and $\left(Q P_{2}\right)_{n}^{G L_{2}}=0$.

Now, we consider the degree $n=2^{s+t}+2^{s}-2$ with $s, t$ non-negative integers, $t \geqslant 2$. Since $\left(\widetilde{S q}_{*}^{0}\right)^{s}:\left(Q P_{2}\right)_{n} \rightarrow\left(Q P_{2}\right)_{2^{t}-1}$ is a isomorphism of $G L_{2}$-modules, we need only to compute $\left(Q P_{2}\right)_{2^{t}-1}^{G L_{2}}$. According to Peterson [17], $\left(Q P_{2}\right)_{2^{t}-1}$ is the vector space of dimension 3 with a basis consisting of 3 classes represented by the following monomials:

$$
u_{t, 1}=x_{1}^{2^{t}-1}, u_{t, 2}=x_{2}^{2^{t}-1}, u_{t, 3}=x_{1} x_{2}^{2^{t}-2} .
$$

Suppose $\theta_{t}=a_{1} u_{t, 1}+a_{2} u_{t, 2}+a_{3} u_{t, 3}$ with $a_{1}, a_{2}, a_{3} \in \mathbb{F}_{2}$ and $\left[\theta_{t}\right] \in\left(Q P_{2}\right)_{2^{t}-1}^{G L_{2}}$. By a simple computation, we have $\rho_{1}\left(\theta_{t}\right)=a_{1} u_{t, 2}+a_{2} u_{t, 1}+a_{3} u_{t, 3} \equiv \theta_{t}$, hence 
$a_{1}=a_{2}=a$. Then, $\rho_{2}\left(\theta_{t}\right) \equiv a\left(u_{t, 1}+u_{t, 2}\right)+a u_{t, 2}+a_{3}\left(u_{t, 2}+u_{t, 3}\right) \equiv \theta_{t}$. So, we get $a_{3}=a$. Hence, $\theta_{t}=a p_{2,0, t}$ with $p_{2,0, t}=u_{t, 1}+u_{t, 2}+u_{t, 3}$ and

$$
\left(Q P_{2}\right)_{n}^{G L_{2}}=\left\langle\left[\psi^{s}\left(p_{2,0, t}\right)\right]\right\rangle .
$$

Combining the above results, we obtain

Proposition 3.1.2. Let $n$ be a non-negative integer. We have

$$
\left(Q P_{2}\right)_{n}^{G L_{2}}= \begin{cases}\left\langle\left[p_{2, s}\right]\right\rangle, & \text { if } n=2^{s+1}-2, s \geqslant 0 \\ \left\langle\left[p_{2, s, t}\right]\right\rangle, & \text { if } n=2^{s+t}+2^{s}-2, s \geqslant 0, t \geqslant 2, \\ 0, & \text { otherwise, }\end{cases}
$$

where $p_{2, s, t}=\psi^{s}\left(p_{2,0, t}\right)$.

Recall that $\left(P_{2}\right)^{*}=\Gamma\left(a_{1}, a_{2}\right)$. For any $s, t \geqslant 0$, we set

$$
q_{2, s, t}:=a_{1}^{\left(2^{s}-1\right)} a_{2}^{\left(2^{s+t}-1\right)} \in P\left(\left(P_{2}\right)_{2^{s+t}+2^{s}-2}^{*}\right) .
$$

Since $\left\langle q_{2, s, 0}, p_{2, s}\right\rangle=1$ and $\left\langle q_{2, s, t}, p_{2, s, t}\right\rangle=1$ for every $s \geqslant 0, t \geqslant 2$, from Proposition 3.1.2, we get the following.

Proposition 3.1.3. For $n$ a non-negative integer, we obtain

$$
\mathbb{F}_{2} \otimes_{G L_{2}} P\left(\left(P_{2}\right)_{n}^{*}\right)= \begin{cases}\left\langle\left[q_{2, s, 0}\right]\right\rangle, & \text { if } n=2^{s+1}-2, s \geqslant 0 \\ \left\langle\left[q_{2, s, t}\right]\right\rangle, & \text { if } n=2^{s+t}+2^{s}-2, s \geqslant 0, t \geqslant 2, \\ 0, & \text { otherwise. }\end{cases}
$$

It is easy to see that $\phi_{2}\left(q_{2, s, t}\right)=\lambda_{2^{s}-1} \lambda_{2^{s+t}-1}$ is a cycle in $\Lambda^{2, *}$. Applying Theorem 2.2, we get

$$
\operatorname{Tr}_{2}\left(\left[q_{2, s, t}\right]\right)=\left[\phi_{2}\left(q_{2, s, t}\right)\right]=\left[\lambda_{2^{s}-1} \lambda_{s+t}\right]=h_{s} h_{s+t} .
$$

Since $h_{s} h_{s+1}=0$, applying Theorem 2.1 we have

$$
\operatorname{Ext}_{\mathcal{A}}^{2, m}\left(\mathbb{F}_{2}, \mathbb{F}_{2}\right)= \begin{cases}\left\langle h_{s}^{2}\right\rangle, & \text { if } m=2^{s+1}, \text { with } s \geqslant 0, \\ \left\langle h_{s} h_{s+t}\right\rangle, & \text { if } m=2^{s+t}+2^{s}, \text { with } s \geqslant 0, t \geqslant 2, \\ 0, & \text { otherwise. }\end{cases}
$$

Theorem 3.1.1 is completely proved.

\subsection{Determination of $\mathrm{Tr}_{3}$.}

In this subsection, we present the proof of the following.

Theorem 3.2.1 (Boardman [3). The third Singer algebraic transfer

$$
\operatorname{Tr}_{3}: \mathbb{F}_{2} \otimes_{G L_{3}} P\left(\left(P_{3}\right)^{*}\right) \longrightarrow \operatorname{Ext}_{\mathcal{A}}^{3, *+3}\left(\mathbb{F}_{2}, \mathbb{F}_{2}\right)
$$

is an isomorphism.

To prove this theorem, Boardman [3] computed the space $Q P_{3}^{G L_{3}}$ by using a basis consisting of the all the classes represented by certain polynomials in $P_{3}$. It is difficult to use his method for $k=4$, where there are 315 polynomials instead of 21. We also compute this space, however we use the admissible monomial basis for $Q P_{3}$ that is different from the one of Boardman in 3. Our approach can be apply for $k=4$ by using the admissible monomial basis for $Q P_{4}$ which is given in [21, 23].

From a result of Wood [26], we need only to compute $Q P_{3}^{G L_{3}}$ in the degree $n$ with $\mu(n) \leqslant 3$. 
3.2.1. The case $n=2^{t+1}-2$.

According to Kameko [13], $\left(Q P_{3}\right)_{n}$ is a vector space with a basis consisting of all the classes represented by the following monomials:

$$
\begin{aligned}
& v_{t, 1}=x_{2}^{2^{t}-1} x_{3}^{2^{t}-1}, v_{t, 2}=x_{1}^{2^{t}-1} x_{3}^{2^{t}-1}, v_{t, 3}=x_{1}^{2^{t}-1} x_{2}^{2^{t}-1}, \text { for } t \geqslant 1, \\
& v_{t, 4}=x_{1} x_{2}^{2^{t}-2} x_{3}^{2^{t}-1}, v_{t, 5}=x_{1} x_{2}^{2^{t}-1} x_{3}^{2^{t}-2}, v_{t, 6}=x_{1}^{2^{t}-1} x_{2} x_{3}^{2^{t}-2}, \text { for } t \geqslant 2, \\
& v_{t, 7}=x_{1}^{3} x_{2}^{2^{t}-3} x_{3}^{2^{t}-2}, \text { for } t \geqslant 3
\end{aligned}
$$

Set $p_{3, t}=\sum_{i=1}^{7} v_{t, i}$, with $t \geqslant 3$. By a direct computation, we have

Proposition 3.2.2. For any non-negative integer $t$, we have

$$
\left(Q P_{3}\right)_{2^{t+1}-2}^{G L_{3}}= \begin{cases}\langle 1\rangle, & \text { if } t=0, \\ 0, & \text { if } t=1,2, \\ \left\langle\left[p_{3, t}\right]\right\rangle, & \text { if } t \geqslant 3\end{cases}
$$

Recall that $\left(P_{3}\right)^{*}=\Gamma\left(a_{1}, a_{2}, a_{3}\right)$. We set

$$
q_{3, t}=a_{1}^{(0)} a_{2}^{\left(2^{t}-1\right)} a_{3}^{\left(2^{t}-1\right)} \in P\left(\left(P_{3}\right)_{2^{t+1}-2}^{*}\right) .
$$

Since $\left\langle p_{3, t}, q_{3, t}\right\rangle=1$, we get

$$
\mathbb{F}_{2} \otimes_{G L_{3}} P\left(\left(P_{3}\right)_{2^{t+1}-2}^{*}\right)= \begin{cases}\langle[1]\rangle, & \text { if } t=0 \\ 0, & \text { if } t=1,2, \\ \left\langle\left[q_{3, t}\right]\right\rangle, & \text { if } t \geqslant 3\end{cases}
$$

It is easy to see that $\phi_{3}\left(q_{3, t}\right)=\lambda_{0} \lambda_{2^{t}-1}^{2}$ is a cycle in $\Lambda^{3, *}$. By Theorem 2.2. we have

$$
\operatorname{Tr}_{3}\left(\left[q_{3, t}\right]\right)=\left[\phi_{3}\left(q_{3, t}\right)\right]=\left[\lambda_{0} \lambda_{2^{t}-1}^{2}\right]=h_{0} h_{t}^{2}
$$

According to Theorem 2.1] we have

$$
\operatorname{Ext}_{\mathcal{A}}^{3,2^{t+1}+1}\left(\mathbb{F}_{2}, \mathbb{F}_{2}\right)=\left\langle h_{0} h_{t}^{2}\right\rangle .
$$

Since $h_{0} h_{1}=0$ and $h_{0} h_{2}^{2}=0$, from the above equalities we see that Theorem 3.2 .1 is true in this case.

3.2.2. The case $n=2^{t+u}+2^{u}-3$.

If $u>1$ then $\mu(n)=3$, hence the iterated Kameko homomorphism

$$
\left(\widetilde{S}_{*}^{0}\right)^{u-1}:\left(Q P_{3}\right)_{2^{t+u}+2^{u}-3} \rightarrow\left(Q P_{3}\right)_{2^{t+1}-1}
$$

is also an isomorphism $G L_{3}$-modules. Hence, we need only to compute $\left(Q P_{3}\right)_{2^{t+1}-1}^{G L_{3}}$. According to Kameko [13], $\left(Q P_{3}\right)_{n}$ is a vector space with a basis consisting of all 
the classes represented by the following monomials:

$$
\begin{aligned}
& u_{t, 1}=x_{3}^{2^{t+1}-1}, u_{t, 2}=x_{2}^{2^{t+1}-1}, u_{t, 3}=x_{1}^{2^{t+1}-1}, \text { for } t \geqslant 0, \\
& u_{t, 4}=x_{2} x_{3}^{2^{t+1}-2}, u_{t, 5}=x_{1} x_{3}^{2^{t+1}-2}, u_{t, 6}=x_{1} x_{2}^{2^{t+1}-2}, \text { for } t \geqslant 1, \\
& u_{1,7}=x_{1} x_{2} x_{3}, \text { for } t=1, \\
& u_{t, 7}=x_{1} x_{2}^{2} x_{3}^{2^{t+1}-4}, u_{t, 8}=x_{1} x_{2}^{2^{t}-1} x_{3}^{2^{t}-1}, \\
& u_{t, 9}=x_{1}^{2^{t}-1} x_{2} x_{3}^{2^{t}-1}, u_{t, 10}=x_{1}^{2^{t}-1} x_{2}^{2^{t}-1} x_{3}, \text { for } t \geqslant 2, \\
& u_{t, 11}=x_{1}^{3} x_{2}^{2^{t}-3} x_{3}^{2^{t}-1}, u_{t, 12}=x_{1}^{3} x_{2}^{2^{t}-1} x_{3}^{2^{t}-3}, u_{t, 13}=x_{1}^{2^{t}-1} x_{2}^{3} x_{3}^{2^{t}-3}, \text { for } t \geqslant 3, \\
& u_{t, 14}=x_{1}^{7} x_{2}^{2^{t}-5} x_{3}^{2^{t}-3}, \text { for } t \geqslant 4 .
\end{aligned}
$$

Set $p_{3, t, 1}=\sum_{i=1}^{7} u_{t, i}$ for $t \geqslant 1$ and $\bar{p}_{3, t, 1}=\sum_{j=7}^{14} u_{t, j}$ for $t \geqslant 4$. By a direct computation we have

Proposition 3.2.3. For any integers $t \geqslant 0, u>0$, we have

$$
\left(Q P_{3}\right)_{2^{t+u}+2^{u}-3}^{G L_{3}}= \begin{cases}0, & \text { if } t=0, \\ \left\langle\left[p_{3, t, u}\right]\right\rangle, & \text { if } 1 \leqslant t \leqslant 3, \\ \left\langle\left[p_{3, t, u}\right],\left[\bar{p}_{3, t, u}\right]\right\rangle, & \text { if } t \geqslant 4,\end{cases}
$$

where $p_{3, t, u}=\psi^{u-1}\left(p_{3, t, 1}\right), \bar{p}_{3, t, u}=\psi^{u-1}\left(\bar{p}_{3, t, 1}\right)$.

We set

$$
q_{3, t, u}=a_{1}^{\left(2^{u-1}-1\right)} a_{2}^{\left(2^{u-1}-1\right)} a_{3}^{\left(2^{t+u}-1\right)}, \quad \bar{q}_{3, t, u}=a_{1}^{\left(2^{u}-1\right)} a_{2}^{\left(2^{t+u-1}-1\right)} a_{3}^{\left(2^{t+u-1}-1\right)} .
$$

It is easy to see that $q_{3, t, u}, \bar{q}_{3, t, u} \in P\left(\left(P_{3}\right)_{2^{t+1}-2}^{*}\right)$ and

$$
\begin{aligned}
& \left\langle p_{3, t, u}, q_{3, t, u}\right\rangle=1,\left\langle p_{3, t, u}, \bar{q}_{3, t, u}\right\rangle=0, \\
& \left\langle\bar{p}_{3, t, u}, q_{3, t, u}\right\rangle=0,\left\langle\bar{p}_{3, t, u}, \bar{q}_{3, t, u}\right\rangle=1 .
\end{aligned}
$$

So, we get

$$
\mathbb{F}_{2} \otimes_{G L_{3}} P\left(\left(P_{3}\right)_{2^{t+u}+2^{u}-3}^{*}\right)= \begin{cases}0, & \text { if } t=0, \\ \left\langle\left[q_{3, t, u}\right]\right\rangle, & \text { if } 1 \leqslant t \leqslant 3, \\ \left\langle\left[q_{3, t, u}\right],\left[\bar{q}_{3, t, u}\right]\right\rangle, & \text { if } t \geqslant 4 .\end{cases}
$$

By applying Theorem 2.2, we have

$$
\begin{aligned}
& \phi_{3}\left(q_{3, t, u}\right)=\lambda_{2^{u-1}-1}^{2} \lambda_{2^{t+u}-1}, \\
& \phi_{3}\left(\bar{q}_{3, t, u}\right)=\lambda_{2^{u}-1} \lambda_{2^{t+u-1}-1}^{2}
\end{aligned}
$$

are the cycles in $\Lambda^{3, *}$. So, we obtain

$$
\begin{aligned}
& \operatorname{Tr}_{3}\left(\left[q_{3, t, u}\right]\right)=\left[\phi_{3}\left(q_{3, t, u}\right)\right]=\left[\lambda_{2^{u-1}-1}^{2} \lambda_{2^{t+u}-1}\right]=h_{u-1}^{2} h_{t+u}, \\
& \operatorname{Tr}_{3}\left(\left[\bar{q}_{3, t, u}\right]\right)=\left[\phi_{3}\left(\bar{q}_{3, t, u}\right)\right]=\left[\lambda_{2^{u}-1}^{2} \lambda_{2^{t+u-1}-1}^{2}\right]=h_{u} h_{t+u-1}^{2} .
\end{aligned}
$$

According to Theorem 2.1] we have

$$
\operatorname{Ext}_{\mathcal{A}}^{3,2^{t+u}+2^{u}}\left(\mathbb{F}_{2}, \mathbb{F}_{2}\right)=\left\langle h_{u} h_{t+u-1}^{2}, h_{u-1}^{2} h_{t+u}\right\rangle .
$$

If $t=0$ then $h_{u} h_{u-1}^{2}=h_{u}^{2} h_{u-1}=0$. If $t=1$ then $h_{u} h_{t+u-1}^{2}=h_{u}^{3}=h_{u-1}^{2} h_{u+1}=$ $h_{u-1}^{2} h_{t+u}$. If $t=2$ then $h_{u} h_{t+u-1}^{2}=h_{u} h_{u+1}^{2}=0$. If $t=3$ then $h_{u} h_{t+u-1}^{2}=$ $h_{u} h_{u+2}^{2}=0$. Hence, from the above equalities we can easily see that Theorem 3.2 .1 is true in this case. 
3.2.3. The case $n=2^{s+u+1}+2^{u+1}+2^{u}-3$.

If $u>0$ then $\mu(n)=3$, hence the iterated Kameko homomorphism

$$
\left(\widetilde{S q}_{*}^{0}\right)^{u}:\left(Q P_{3}\right)_{2^{s+u}+2^{u}-3} \rightarrow\left(Q P_{3}\right)_{2^{s+1}}
$$

is also an isomorphism of $G L_{3}$-modules. Hence, we need only to compute $\left(Q P_{3}\right)_{2^{s+1}}^{G L_{3}}$.

According to Kameko [13], $\left(Q P_{3}\right)_{2^{s+1}}$ is a vector space with a basis consisting of all the classes represented by the following monomials:

$$
\begin{aligned}
& v_{s, 1}=x_{2} x_{3}^{2^{s+1}-1}, v_{s, 2}=x_{2}^{2^{s+1}-1} x_{3}, v_{s, 3}=x_{1} x_{3}^{2^{s+1}-1}, \\
& v_{s, 4}=x_{1} x_{2}^{2^{s+1}-1}, v_{s, 5}=x_{1}^{2^{s+1}-1} x_{3}, v_{s, 6}=x_{1}^{2^{s+1}-1} x_{2}, \text { for } s \geqslant 1, \\
& v_{1,7}=x_{1} x_{2} x_{3}^{2}, v_{1,8}=x_{1} x_{2}^{2} x_{3}, \text { for } s=1, \\
& v_{s, 7}=x_{2}^{3} x_{3}^{2^{s+1}-3}, v_{s, 8}=x_{1}^{3} x_{3}^{2^{s+1}-3}, v_{s, 9}=x_{1}^{3} x_{2}^{2^{s+1}-3}, \\
& v_{s, 10}=x_{1} x_{2} x_{2}^{2^{s+1}-2}, v_{s, 11}=x_{1} x_{2}^{2^{s+1}-2} x_{3}, v_{s, 12}=x_{1} x_{2}^{2} x_{3}^{2^{s+1}-3} \\
& v_{s, 13}=x_{1} x_{2}^{3} x_{3}^{2^{s+1}-4}, v_{s, 14}=x_{1}^{3} x_{2} x_{3}^{2^{s+1}-4} \text { for } s \geqslant 2 \\
& v_{15}=x_{1}^{3} x_{2}^{4} x_{3}, \text { for } s=2 .
\end{aligned}
$$

Set $\bar{p}_{0}=v_{2,10}+v_{2,11}+v_{2,14}+v_{2,15}$. By a direct computation, we have

Proposition 3.2.4. For any integers $s>0, u \geqslant 0$ and $n=2^{s+u+1}+2^{u+1}+2^{u}-3$, we have

$$
\left(Q P_{3}\right)_{n}^{G L_{3}}= \begin{cases}\left\langle\left[\psi^{u}\left(\bar{p}_{0}\right)\right]\right\rangle, & \text { if } s=2, \\ 0, & \text { if } s \neq 2\end{cases}
$$

We set

$$
\begin{aligned}
\bar{c}_{u}= & a_{1}^{\left(3.2^{u}-1\right)} a_{2}^{\left(4.2^{u}-1\right)} a_{3}^{\left(4.2^{u}-1\right)}+a_{1}^{\left(2.2^{u}-1\right)} a_{2}^{\left(5.2^{u}-1\right)} a_{3}^{\left(4.2^{u}-1\right)} \\
& +a_{1}^{\left(2.2^{u}-1\right)} a_{2}^{\left(3.2^{u}-1\right)} a_{3}^{\left(6.2^{u}-1\right)}+a_{1}^{\left(2.2^{u}-1\right)} a_{2}^{\left(2.2^{u}-1\right)} a_{3}^{\left(7.2^{u}-1\right)}
\end{aligned}
$$

is an element in $\left(P_{3}\right)^{*}=\Gamma\left(a_{1}, a_{2}, a_{3}\right)$. By a direct computation, we can see that $\bar{c}_{u} \in P\left(\left(P_{3}\right)_{2^{t+u}+2^{u}-3}^{*}\right)$ and $\left\langle\psi^{u}\left(\bar{p}_{0}\right), \bar{c}_{u}\right\rangle=1$. So, we get

$$
\mathbb{F}_{2} \otimes_{G L_{3}} P\left(\left(P_{3}\right)_{n}^{*}\right)= \begin{cases}\left\langle\left[\bar{c}_{u}\right]\right\rangle, & \text { if } s=2, \\ 0, & \text { if } s \neq 2 .\end{cases}
$$

For $u=0$, we have $\bar{c}_{0}=a_{1}^{(2)} a_{2}^{(3)} a_{3}^{(3)}+a_{1}^{(1)} a_{2}^{(4)} a_{3}^{(3)}+a_{1}^{(1)} a_{2}^{(2)} a_{3}^{(5)}+a_{1}^{(1)} a_{2}^{(1)} a_{3}^{(6)}$.

A direct computation shows

$$
\begin{aligned}
& \phi_{3}\left(a_{1}^{(2)} a_{2}^{(3)} a_{3}^{(3)}\right)=\lambda_{2} \lambda_{3}^{2}+\lambda_{1} \lambda_{4} \lambda_{3}+\lambda_{1} \lambda_{3} \lambda_{4}, \\
& \phi_{3}\left(a_{1}^{(1)} a_{2}^{(4)} a_{3}^{(3)}\right)=\lambda_{1} \lambda_{4} \lambda_{3}+\lambda_{1} \lambda_{3} \lambda_{4}+\lambda_{1} \lambda_{2} \lambda_{5}, \\
& \phi_{3}\left(a_{1}^{(1)} a_{2}^{(2)} a_{3}^{(5)}\right)=\lambda_{1} \lambda_{2} \lambda_{5}+\lambda_{1}^{2} \lambda_{6}, \\
& \phi_{3}\left(a_{1}^{(1)} a_{2}^{(1)} a_{3}^{(6)}\right)=\lambda_{1}^{2} \lambda_{6} .
\end{aligned}
$$

Hence, we obtain $\phi_{3}\left(\bar{c}_{0}\right)=\lambda_{2} \lambda_{3}^{2}$. By Theorem 2.2 we have $\operatorname{Tr}_{3}\left(\left[\bar{c}_{0}\right]\right)=\left[\lambda_{2} \lambda_{3}^{2}\right]=c_{0}$. Since $\left[\bar{c}_{u}\right]=\left(\widetilde{S q}_{*}^{0}\right)^{u}\left(\left[\bar{c}_{0}\right]\right)$, we get

$$
\operatorname{Tr}_{3}\left(\left[\bar{c}_{u}\right]\right)=\operatorname{Tr}_{3}\left(\left(\widetilde{S q}^{0}\right)^{u}\left(\left[\bar{c}_{0}\right]\right)\right)=\left(S q^{0}\right)^{u} \operatorname{Tr}_{3}\left(\left[\bar{c}_{0}\right]\right)=\left(S q^{0}\right)^{u}\left(c_{0}\right)=c_{u}
$$


By Theorem 2.1 we have $h_{u} h_{u+1}=0$. Hence,

$$
\operatorname{Ext}_{\mathcal{A}}^{3,2^{s+u+1}+2^{u+1}+2^{u}}\left(\mathbb{F}_{2}, \mathbb{F}_{2}\right)= \begin{cases}\left\langle h_{u} h_{u+1} h_{u+3}, c_{u}\right\rangle=\left\langle c_{u}\right\rangle, & \text { if } s=2, \\ \left\langle h_{u} h_{u+1} h_{s+u+1}\right\rangle=0, & \text { if } s \neq 2 .\end{cases}
$$

Theorem 3.2.1 in this case follows from the above equalities.

\subsubsection{The case of the generic degree.}

In this subsection, we consider the degree

$$
n=2^{s+t+u}+2^{t+u}+2^{u}-3,
$$

with $s, t, u$ non-negative integers.

The subcases either $s=0$ or $t=0$ have been determined in Subsections 3.2 .1 and 3.2.2. The case $s>0$ and $t=1$ has been determined in Subsection 3.2.3. So, we assume that $s>0$ and $t>1$.

The iterated homomorphism

$$
\left(\widetilde{S q}_{*}^{0}\right)^{u}:\left(Q P_{3}\right)_{2^{s+t+u}+2^{t+u}+2^{u}-3} \rightarrow\left(Q P_{3}\right)_{2^{s+t}+2^{t}-2}
$$

is an isomorphism of $G L_{3}$-modules. So, we need only to compute $\left(Q P_{3}\right)_{2^{s+t}+2^{t}-2}^{G L_{3}}$.

The subcase $s=1$. Then $n=2^{t+1}+2^{t}-2$. According to Kameko [13], $\left(Q P_{3}\right)_{n}$ is the vector space with a basis consisting of all the classes represented by the following monomials:

$$
\begin{array}{cll}
v_{t, 1}=x_{2}^{2^{t}-1} x_{3}^{2^{t+1}-1} & v_{t, 2}=x_{2}^{2^{t+1}-1} x_{3}^{2^{t}-1} & v_{t, 3}=x_{1}^{2^{t}-1} x_{3}^{2^{t+1}-1} \\
v_{t, 4}=x_{1}^{2^{t}-1} x_{2}^{2^{t+1}-1} & v_{t, 5}=x_{1}^{2^{t+1}-1} x_{3}^{2^{t}-1} & v_{t, 6}=x_{1}^{2^{t+1}-1} x_{2}^{2^{t}-1} \\
v_{t, 7}=x_{1} x_{2}^{2^{t}-2} x_{3}^{2^{t+1}-1} & v_{t, 8}=x_{1} x_{2}^{2^{t+1}-1} x_{3}^{2^{t}-2} & v_{t, 9}=x_{1}^{2^{t+1}-1} x_{2} x_{3}^{2^{t}-2} \\
v_{t, 10}=x_{1} x_{2}^{2^{t}-1} x_{3}^{2^{t+1}-2} & v_{t, 11}=x_{1} x_{2}^{2^{t+1}-2} x_{3}^{2^{t}-1} & v_{t, 12}=x_{1}^{2^{t}-1} x_{2} x_{3}^{2^{t+1}-2} \\
v_{t, 13}=x_{1}^{3} x_{2}^{2^{t+1}-3} x_{3}^{2^{t}-2}, & \\
v_{2,14}=x_{1}^{3} x_{2}^{3} x_{3}^{4} \text { for } t=2, \text { and } & v_{t, 14}=x_{1}^{3} x_{2}^{2^{t}-3} x_{3}^{2^{t+1}-2} & \text { for } t>2 .
\end{array}
$$

By a direct computation using the above basis, we obtain

Proposition 3.2.5. For any integers $t>1, u \geqslant 0$ and $n=2^{t+u+1}+2^{t+u}+2^{u}-3$, we have $\left(Q P_{3}\right)_{n}^{G L_{3}}=0$.

By Theorem $2.1 h_{t+u} h_{t+u+1}=0$, so we have

$$
\operatorname{Ext}_{\mathcal{A}}^{3,2^{t+u+1}+2^{t+u}+2^{u}}\left(\mathbb{F}_{2}, \mathbb{F}_{2}\right)=\left\langle h_{u} h_{t+u} h_{t+u+1}\right\rangle=0 .
$$

Hence, from the above equalities, we can see that

$$
\operatorname{Tr}_{3}: \mathbb{F}_{2} \otimes_{G L_{3}} P\left(\left(P_{3}\right)_{2^{t+u+1}+2^{t+u}+2^{u}-3}^{*}\right) \longrightarrow \operatorname{Ext}_{\mathcal{A}}^{3,2^{t+u+1}+2^{t+u}+2^{u}}\left(\mathbb{F}_{2}, \mathbb{F}_{2}\right)
$$

is a trivial isomorphism.

Now, suppose that $s, t>1$ and $n=2^{s+t}+2^{t}-2$. From the results of Kameko [13, we see that $\left(Q P_{3}\right)_{n}$ is the vector space of dimension 21 with a basis consisting of all the classes represented by the following monomials: 


$$
\begin{array}{ll}
v_{s, t, 1}=x_{2}^{2^{t}-1} x_{3}^{2^{s+t}-1} & v_{s, t, 2}=x_{2}^{2^{s+t}-1} x_{3}^{2^{t}-1} \\
v_{s, t, 3}=x_{1}^{2^{t}-1} x_{3}^{2^{s+t}-1} & v_{s, t, 4}=x_{1}^{2^{t}-1} x_{2}^{2^{s+t}-1} \\
v_{s, t, 5}=x_{1}^{2^{s+t}-1} x_{3}^{2^{t}-1} & v_{s, t, 6}=x_{1}^{2^{s+t}-1} x_{2}^{2^{t}-1} \\
v_{s, t, 7}=x_{2}^{2^{t+1}-1} x_{3}^{2^{s+t}-2^{t}-1} & v_{s, t, 8}=x_{1}^{2^{t+1}-1} x_{3}^{2^{s+t}-2^{t}-1} \\
v_{s, t, 9}=x_{1}^{2^{t+1}-1} x_{2}^{2^{s+t}-2^{t}-1} & v_{s, t, 10}=x_{1} x_{2}^{2^{t}-2} x_{3}^{2^{s+t}-1} \\
v_{s, t, 11}=x_{1} x_{2}^{2^{s+t}-1} x_{3}^{2^{t}-2} & v_{s, t, 12}=x_{1}^{2^{s+t}-1} x_{2} x_{3}^{2^{t}-2} \\
v_{s, t, 13}=x_{1} x_{2}^{2^{t}-1} x_{3}^{2^{s+t}-2} & v_{s, t, 14}=x_{1} x_{2}^{2^{s+t}-2} x_{3}^{2^{t}-1} \\
v_{s, t, 15}=x_{1}^{2^{t}-1} x_{2} x_{3}^{2^{s+t}-2} & v_{s, t, 16}=x_{1} x_{2}^{2^{t+1}-2} x_{3}^{2^{s+t}-2^{t}-1} \\
v_{s, t, 17}=x_{1} x_{2}^{2^{t+1}-1} x_{3}^{2^{s+t}-2^{t}-2} & v_{s, t, 18}=x_{1}^{2^{t+1}-1} x_{2} x_{3}^{2^{s+t}-2^{t}-2} \\
v_{s, t, 19}=x_{1}^{3} x_{2}^{2^{s+t}-3} x_{3}^{2^{t}-2} & v_{s, t, 20}=x_{1}^{3} x_{2}^{2^{t+1}-3} x_{3}^{2^{s+t}-2^{t}-2}, \\
v_{s, 2,21}=x_{1}^{3} x_{2}^{3} x_{3}^{2^{s+2}-4}, \text { for } t=2 \text { and } v_{s, t, 21}=x_{1}^{3} x_{2}^{2^{t}-3} x_{3}^{2^{s+t}-2} \text { for } t>2 .
\end{array}
$$

We set

$$
p_{3, s, t, u}= \begin{cases}\sum_{1 \leqslant j \leqslant 21, j \neq 13,15} \psi^{u}\left(v_{s, 2, j}\right), & \text { if } t=2, \\ \sum_{1 \leqslant j \leqslant 21} \psi^{u}\left(v_{s, t, j}\right), & \text { if } t>2 .\end{cases}
$$

By a direct computation using this basis, we get

Proposition 3.2.6. For any integers $s, t>1, u \geqslant 0$ and $n=2^{s+t+u}+2^{t+u}+2^{u}-3$, we have $\left(Q P_{3}\right)_{n}^{G L_{3}}=\left\langle\left[p_{3, s, t, u}\right]\right\rangle$.

By Theorem 2.1, we have

$$
\operatorname{Ext}_{\mathcal{A}}^{3,2^{s+t+u}+2^{t+u}+2^{u}}\left(\mathbb{F}_{2}, \mathbb{F}_{2}\right)=\left\langle h_{u} h_{t+u} h_{s+t+u}\right\rangle
$$

Note that $\psi^{u}\left(v_{s, t, 1}\right)=x_{1}^{2^{u}-1} x_{2}^{2^{t+u}-1} x_{3}^{2^{s+t+u}-1}$. Consider the element

$$
q_{3, s, t, u}=a_{1}^{\left(2^{u}-1\right)} a_{2}^{\left(2^{t+u}-1\right)} a_{3}^{\left(2^{s+t+u}-1\right)} \in \mathbb{F}_{2} \otimes_{G L_{3}} P\left(\left(P_{3}\right)_{n}^{*}\right) .
$$

Since $\left\langle p_{3, s, t, u}, q_{3, s, t, u}\right\rangle=1$, from Proposition 3.2.6. we obtain

$$
\mathbb{F}_{2} \otimes_{G L_{3}} P\left(\left(P_{3}\right)_{n}^{*}\right)=\left\langle\left[q_{3, s, t, u}\right]\right\rangle
$$

It is easy to see that $\phi_{3}\left(q_{3, s, t, u}\right)=\lambda_{u} \lambda_{t+u} \lambda_{s+t+u}$, hence using Theorem 2.2 we get

$$
\operatorname{Tr}_{3}\left(\left[q_{3, s, t, u}\right]\right)=\left[\lambda_{u} \lambda_{t+u} \lambda_{s+t+u}\right]=h_{u} h_{t+u} h_{s+t+u}
$$

Theorem 3.2 .1 is completely proved.

\section{Determination of $\mathrm{Tr}_{4}$ in some internal Degrees}

In this section, we explicitly determined $T r_{4}$ in some internal degrees. Our main result is the following.

Theorem 4.1. Let $s$ be a positive integer and let $n$ be one of the degrees $2^{s+1}-1$, $2^{s+1}-2,2^{s+1}-3$. If $n \neq 61$ and $n \neq 126$, then the homomorphism

$$
\operatorname{Tr}_{4}: \mathbb{F}_{2} \otimes_{G L_{4}} P\left(\left(P_{4}\right)_{n}^{*}\right) \longrightarrow \operatorname{Ext}_{\mathcal{A}}^{4, n+4}\left(\mathbb{F}_{2}, \mathbb{F}_{2}\right)
$$

is an isomorphism. If either $n=61$ or $n=126$, then $\operatorname{Tr}_{4}$ is a monomorphism but it is not an epimorphism.

We prove the theorem by computing the space $\left(Q P_{4}\right)_{n}^{G L_{4}}$. 
4.1. The case $n=2^{s+1}-3$.

Proposition 4.1.1 (see [20, 23]). Let $n=2^{s+1}-3$ with $s$ a positive integer. Then, the dimension of the $\mathbb{F}_{2}$-vector space $\left(Q P_{4}\right)_{n}$ is determined by the following table:

$$
\begin{array}{c|cccc}
n=2^{s+1}-3 & s=1 & s=2 & s=3 & s \geqslant 4 \\
\hline \operatorname{dim}\left(Q P_{4}\right)_{n} & 4 & 15 & 35 & 45
\end{array}
$$

A basis for $\left(Q P_{4}\right)_{n}$ is the set consisting of all the classes represented monomials $a_{j}=a_{s, j}$ which are determined as follows:

For $s=1, a_{1,1}=x_{4}, a_{1,2}=x_{3}, a_{1,3}=x_{2}, a_{1,4}=x_{1}$.

For $s \geqslant 2$,

$$
\begin{array}{ll}
a_{s, 1}=x_{2}^{2^{s-1}-1} x_{3}^{2^{s-1}-1} x_{4}^{2^{s}-1} & a_{s, 2}=x_{2}^{2^{s-1}-1} x_{3}^{2^{s}-1} x_{4}^{2^{s-1}-1} \\
a_{s, 3}=x_{2}^{2^{s}-1} x_{3}^{2^{s-1}-1} x_{4}^{2^{s-1}-1} & a_{s, 4}=x_{1}^{2^{s-1}-1} x_{3}^{2^{s-1}-1} x_{4}^{2^{s}-1} \\
a_{s, 5}=x_{1}^{2^{s-1}-1} x_{3}^{2^{s}-1} x_{4}^{2^{s-1}-1} & a_{s, 6}=x_{1}^{2^{s-1}-1} x_{2}^{2^{s-1}-1} x_{4}^{2^{s}-1} \\
a_{s, 7}=x_{1}^{2^{s-1}-1} x_{2}^{2^{s-1}-1} x_{3}^{2^{s}-1} & a_{s, 8}=x_{1}^{2^{s-1}-1} x_{2}^{2^{s}-1} x_{4}^{2^{s-1}-1} \\
a_{s, 9}=x_{1}^{2^{s-1}-1} x_{2}^{2^{s}-1} x_{3}^{2^{-1}-1} & a_{s, 10}=x_{1}^{2^{s}-1} x_{3}^{2^{s-1}-1} x_{4}^{2^{s-1}-1} \\
a_{s, 11}=x_{1}^{2^{s}-1} x_{2}^{2^{s-1}-1} x_{4}^{2^{s-1}-1} & a_{s, 12}=x_{1}^{2^{s}-1} x_{2}^{2^{s-1}-1} x_{3}^{2^{s-1}-1}
\end{array}
$$

For $s=2, a_{2,13}=x_{1} x_{2} x_{3} x_{4}^{2}, a_{2,14}=x_{1} x_{2} x_{3}^{2} x_{4}, a_{2,15}=x_{1} x_{2}^{2} x_{3} x_{4}$.

For $s \geqslant 3$,

$$
\begin{aligned}
& \begin{array}{l}
a_{s, 13}=x_{1} x_{2}^{2^{s-1}-2} x_{3}^{2^{s-1}-1} x_{4}^{2^{s}-1} \\
a_{s, 15}=x_{1} x_{2}^{2^{s-1}-1} x_{3}^{2^{s-1}-2} x_{4}^{2^{s}-1}
\end{array} \\
& a_{s, 17}=x_{1} x_{2}^{2^{s}-1} x_{3}^{2^{s-1}-2} x_{4}^{2^{s-1}-1} \\
& a_{s, 19}=x_{1}^{2^{s-1}-1} x_{2} x_{3}^{2^{s-1}-2} x_{4}^{2^{s}-1} \\
& a_{s, 21}=x_{1}^{2^{s-1}-1} x_{2}^{2^{s}-1} x_{3} x_{4}^{2^{s-1}-2} \\
& a_{s, 23}=x_{1}^{2^{s}-1} x_{2} x_{3}^{2^{s-1}-1} x_{4}^{2^{s-1}-2} \\
& a_{s, 25}=x_{1} x_{2}^{2^{s-1}-1} x_{3}^{2^{s-1}-1} x_{4}^{2^{s}-2} \\
& a_{s, 27}=x_{1} x_{2}^{2^{s}-2} x_{3}^{2^{s-1}-1} x_{4}^{2^{s-1}-1} \\
& a_{s, 29}=x_{1}^{2^{s-1}-1} x_{2} x_{3}^{2^{s}-2} x_{4}^{2^{s-1}-1} \\
& \begin{array}{l}
a_{s, 14}=x_{1} x_{2}^{2^{s-1}-2} x_{3}^{2^{s}-1} x_{4}^{2^{s-1}-1} \\
a_{s, 16}=x_{1} x_{2}^{2^{s-1}-1} x_{3}^{2^{s}-1} x_{4}^{2^{s-1}-2} \\
a_{s, 18}=x_{1} x_{2}^{2^{s}-1} x_{3}^{2^{s-1}-1} x_{4}^{2^{s-1}-2} \\
a_{s, 20}=x_{1}^{2^{s-1}-1} x_{2} x_{3}^{2^{s}-1} x_{4}^{2^{s-1}-2} \\
a_{s, 22}=x_{1}^{2^{s}-1} x_{2} x_{3}^{2^{s-1}-2} x_{4}^{2^{s-1}-1} \\
a_{s, 24}=x_{1}^{2^{s}-1} x_{2}^{2^{s-1}-1} x_{3} x_{4}^{2^{s-1}-2} \\
a_{s, 26}=x_{1} x_{2}^{2^{s-1}-1} x_{3}^{2^{s}-2} x_{4}^{2^{s-1}-1} \\
a_{s, 28}=x_{1}^{2^{s-1}-1} x_{2} x_{3}^{2^{s-1}}-1 x_{4}^{2^{s}-2} \\
a_{s, 30}=x_{1}^{2^{s-1}-1} x_{2}^{2^{s-1}-1} x_{3} x_{4}^{2^{s}-2}
\end{array}
\end{aligned}
$$

For $s=3$,

$$
\begin{array}{ll}
a_{3,31}=x_{1}^{3} x_{2}^{3} x_{3}^{5} x_{4}^{2} & a_{3,32}=x_{1}^{3} x_{2}^{5} x_{3}^{2} x_{4}^{3} \quad a_{3,33}=x_{1}^{3} x_{2}^{5} x_{3}^{3} x_{4}^{2} \\
a_{3,34}=x_{1}^{3} x_{2}^{3} x_{3}^{3} x_{4}^{4} & a_{3,35}=x_{1}^{3} x_{2}^{3} x_{3}^{4} x_{4}^{3}
\end{array}
$$

For $s \geqslant 4$,

$$
\begin{array}{ll}
a_{s, 31}=x_{1}^{3} x_{2}^{2^{s-1}-3} x_{3}^{2^{s-1}-2} x_{4}^{2^{s}-1} & a_{s, 32}=x_{1}^{3} x_{2}^{2^{s-1}-3} x_{3}^{2^{s}-1} x_{4}^{2^{s-1}-2} \\
a_{s, 33}=x_{1}^{3} x_{2}^{2^{s}-1} x_{3}^{2^{s-1}-3} x_{4}^{2^{s-1}-2} & a_{s, 34}=x_{1}^{2^{s}-1} x_{2}^{3} x_{3}^{2^{s-1}-3} x_{4}^{2^{s-1}-2} \\
a_{s, 35}=x_{1}^{3} x_{2}^{2^{s-1}-3} x_{3}^{2^{s-1}-1} x_{4}^{2^{s}-2} & a_{s, 36}=x_{1}^{3} x_{2}^{2^{s-1}-3} x_{3}^{2^{s}-2} x_{4}^{2^{s-1}-1} \\
a_{s, 37}=x_{1}^{3} x_{2}^{2^{s-1}-1} x_{3}^{2^{s-1}-3} x_{4}^{2^{s}-2} & a_{s, 38}=x_{1}^{2^{s-1}-1} x_{2}^{3} x_{3}^{2^{s-1}-3} x_{4}^{2^{s}-2} \\
a_{s, 39}=x_{1}^{3} x_{2}^{2^{s-1}-1} x_{3}^{2^{s}-3} x_{4}^{2^{s-1}-2} & a_{s, 40}=x_{1}^{3} x_{2}^{2^{s}-3} x_{3}^{2^{s-1}-2} x_{4}^{2^{s-1}-1} \\
a_{s, 41}=x_{1}^{3} x_{2}^{2^{s}-3} x_{3}^{2^{s-1}-1} x_{4}^{2^{s-1}-2} & a_{s, 42}=x_{1}^{2^{s-1}-1} x_{2}^{3} x_{3}^{2^{s}-3} x_{4}^{2^{s-1}-2} \\
a_{s, 43}=x_{1}^{7} x_{2}^{2^{s}-5} x_{3}^{2^{s-1}-3} x_{4}^{2^{s-1}-2} &
\end{array}
$$

For $s=4, a_{4,44}=x_{1}^{7} x_{2}^{7} x_{3}^{9} x_{4}^{6}, a_{4,45}=x_{1}^{7} x_{2}^{7} x_{3}^{7} x_{4}^{8}$.

For $s \geqslant 5, a_{s, 44}=x_{1}^{7} x_{2}^{2^{s-1}-5} x_{3}^{2^{s}-3} x_{4}^{2^{s-1}-2}, a_{s, 45}=x_{1}^{7} x_{2}^{2^{s-1}-5} x_{3}^{2^{s-1}-3} x_{4}^{2^{s}-2}$. 
Proposition 4.1.2. Let $s$ be a positive integer. Then, $\left(Q P_{4}\right)_{2^{s+1}-3}^{G L_{4}}=0$.

For simplicity, we prove the proposition in detail for $s \geqslant 5$. The other cases can be proved by the similar computations.

For any monomials $z_{1}, z_{2}, \ldots, z_{m}$ in $P_{k}$ and for a subgroup $G \subset G L_{k}$, we denote $G\left(z_{1}, z_{2}, \ldots, z_{m}\right)$ the $G$-submodule of $Q P_{k}$ generated by the set $\left\{\left[z_{i}\right]: 1 \leqslant i \leqslant m\right\}$. We have the following.

Lemma 4.1.3. i) For any $s \geqslant 5$, there is a direct summand decomposition of the $\Sigma_{4}$-modules:

$$
\left(Q P_{4}\right)_{2^{s+1}-3}=\Sigma_{4}\left(a_{s, 1}\right) \oplus \Sigma_{4}\left(a_{s, 13}\right) \oplus \Sigma_{4}\left(a_{s, 31}\right) \oplus \Sigma_{4}\left(a_{s, 25}, a_{s, 35}, a_{s, 43}\right) .
$$

ii) $\Sigma_{4}\left(a_{s, 1}\right)^{\Sigma_{4}}=\left\langle\left[p_{4, s, 1}\right]\right\rangle$, with $p_{4, s, 1}=\sum_{j=1}^{12} a_{s, j}$.

iii) $\Sigma_{4}\left(a_{s, 13}\right)^{\Sigma_{4}}=\left\langle\left[p_{4, s, 2}\right]\right\rangle$, with $p_{4, s, 2}=\sum_{j=13}^{24} a_{s, j}$.

iv) $\Sigma_{4}\left(a_{s, 31}\right)^{\Sigma_{4}}=\left\langle\left[p_{4, s, 3}\right]\right\rangle$, with $p_{4, s, 3}=\sum_{j=31}^{34} a_{s, j}$.

v) $\Sigma_{4}\left(a_{s, 25}, a_{s, 35}, a_{s, 43}\right)^{\Sigma_{4}}=\left\langle\left[p_{4, s, 4}\right]\right\rangle$, with

$$
p_{4, s, 4}=\sum_{j=25}^{30} a_{s, j}+\sum_{j=39}^{43} a_{s, j}+a_{s, 45} .
$$

Proof. We obtain Part i) by a simple computation using Proposition 4.1.1. We prove Part v) in detail. The others can be proved by the similar computations. By a simple computation we see that the set $\left\{\left[a_{s, j}\right]: j=25, \ldots, 30,35 \ldots, 45\right\}$ is a basis for $\Sigma_{4}\left(a_{s, 25}, a_{s, 35}, a_{s, 43}\right)$. Suppose $[f] \in \Sigma_{4}\left(a_{s, 25}, a_{s, 35}, a_{s, 43}\right)^{\Sigma_{4}}$, then

$$
f \equiv \sum_{j=25}^{30} \gamma_{j} a_{s, j}+\sum_{j=35}^{45} \gamma_{j} a_{s, j}
$$

with $\gamma_{j} \in \mathbb{F}_{2}$. By a direct computation, we get

$$
\begin{aligned}
\rho_{1}(f)+f \equiv & \left(\gamma_{25}+\gamma_{28}\right)\left(a_{s, 25}+a_{s, 28}\right)+\left(\gamma_{26}+\gamma_{29}\right)\left(a_{s, 26}+a_{s, 29}\right) \\
& +\left(\gamma_{27}+\gamma_{41}\right) a_{s, 35}+\left(\gamma_{27}+\gamma_{40}\right) a_{s, 36}+\left(\gamma_{37}+\gamma_{38}\right)\left(a_{s, 37}+a_{s, 38}\right) \\
& +\left(\gamma_{39}+\gamma_{42}\right)\left(a_{s, 39}+a_{s, 42}\right)+\left(\gamma_{41}+\gamma_{43}\right) a_{s, 44}+\left(\gamma_{40}+\gamma_{43}\right) a_{s, 45} \equiv 0, \\
\rho_{2}(f)+f \equiv & \left(\gamma_{26}+\gamma_{27}\right)\left(a_{s, 26}+a_{s, 27}\right)+\left(\gamma_{28}+\gamma_{30}\right)\left(a_{s, 28}+a_{s, 30}\right) \\
& +\left(\gamma_{35}+\gamma_{37}\right)\left(a_{s, 35}+a_{s, 37}\right)+\left(\gamma_{29}+\gamma_{36}+\gamma_{40}\right)\left(a_{s, 36}+a_{s, 40}\right) \\
& +\left(\gamma_{39}+\gamma_{41}\right)\left(a_{s, 39}+a_{s, 41}\right)+\left(\gamma_{42}+\gamma_{43}+\gamma_{44}\right)\left(a_{s, 43}+a_{s, 44}\right) \\
& +\left(\gamma_{29}+\gamma_{42}\right)\left(a_{s, 38}+a_{s, 45}\right) \equiv 0, \\
\rho_{3}(f)+f \equiv & \left(\gamma_{25}+\gamma_{26}\right)\left(a_{s, 25}+a_{s, 26}\right)+\left(\gamma_{28}+\gamma_{29}\right)\left(a_{s, 28}+a_{s, 29}\right) \\
& +\left(\gamma_{35}+\gamma_{36}\right)\left(a_{s, 35}+a_{s, 36}\right)+\left(\gamma_{30}+\gamma_{37}+\gamma_{39}\right)\left(a_{s, 37}+a_{s, 39}\right) \\
& +\left(\gamma_{30}+\gamma_{38}+\gamma_{42}\right)\left(a_{s, 38}+a_{s, 42}\right)+\left(\gamma_{40}+\gamma_{41}\right)\left(a_{s, 40}+a_{s, 41}\right) \\
& +\left(\gamma_{30}+\gamma_{44}+\gamma_{45}\right)\left(a_{s, 44}+a_{s, 45}\right) \equiv 0 .
\end{aligned}
$$

The above equalities imply $\gamma_{j}=0$ for $j=35,36,37,38,44$ and $\gamma_{j}=\gamma_{25}$ for $j \neq$ $35,36,37,38,44$. The lemma is proved.

Proof of Proposition 4.1.2. Let $f \in P_{4}$ such that $[f] \in\left(Q P_{4}\right)_{2^{s+1}-3}^{G L_{4}}$. Since $\Sigma_{4} \subset$ $G L_{4}$, we have $[f] \in\left(Q P_{4}\right)_{2^{s+1}-3}^{\Sigma_{4}}$. Then, $f \equiv \sum_{j=1}^{4} \gamma_{j} p_{4, s, j}$ with $\gamma_{j} \in \mathbb{F}_{2}$. By a 
direct computation, we get

$$
\begin{aligned}
\rho_{4}(f)+f \equiv & \left(\gamma_{1}+\gamma_{4}\right) a_{s, 3}+\gamma_{1} a_{s, 9}+\left(\gamma_{2}+\gamma_{3}\right) a_{s, 15} \\
& +\gamma_{2} a_{s, 21}+\text { other terms } \equiv 0 .
\end{aligned}
$$

The last equality implies $\gamma_{j}=0$ for $j=1,2,3,4$. The proposition follows.

From Theorem 2.1, we see that $\operatorname{Ext}_{\mathcal{A}}^{4,2^{s+1}+1}\left(\mathbb{F}_{2}, \mathbb{F}_{2}\right)=0$ for any $s \neq 5$ and $\operatorname{Ext}_{\mathcal{A}}^{4,65}\left(\mathbb{F}_{2}, \mathbb{F}_{2}\right)=\left\langle D_{3}(0)\right\rangle$. Hence, Theorem 4.1 holds for $n=2^{s+1}-3$.

4.2. The case $n=2^{s+1}-2$.

Since Kameko's homomorphism in the degree $2^{s+1}-2$,

$$
\left(\widetilde{S q}_{*}^{0}\right)_{2^{s+1}-2}:\left(Q P_{4}\right)_{2^{s+1}-2} \rightarrow\left(Q P_{4}\right)_{2^{s}-3}
$$

is an epimorphism of $G L_{4}$-modules, using Proposition 4.1.2, we have

$$
\left(Q P_{4}\right)_{2^{s+1}-2}^{G L_{4}} \subset\left(\operatorname{Ker}\left(\widetilde{S q}_{*}^{0}\right)_{2^{s+1}-2}\right)^{G L_{4}} .
$$

From [20, 23, we have the following.

Proposition 4.2.1 (see [20, 23]). Let $s$ be a positive integer. Then,

$$
\operatorname{dim}\left(\operatorname{Ker}\left(\widetilde{S q}_{*}^{0}\right)_{2^{s+1}-2}\right)= \begin{cases}6, & \text { if } s=1, \\ 20, & \text { if } s=2, \\ 35 & \text { if } s \geqslant 3 .\end{cases}
$$

A basis for $\left(\operatorname{Ker}\left(\widetilde{S q}_{*}^{0}\right)_{2^{s+1}-2}\right)$ is the set consisting of all the classes represented monomials $b_{j}=b_{s, j}$ which are determined as follows:

For $s \geqslant 1$,

$$
\begin{array}{lll}
b_{s, 1}=x_{3}^{2^{s}-1} x_{4}^{2^{s}-1} & b_{s, 2}=x_{2}^{2^{s}-1} x_{4}^{2^{s}-1} & b_{s, 3}=x_{2}^{2^{s}-1} x_{3}^{2^{s}-1} \\
b_{s, 4}=x_{1}^{2^{s}-1} x_{4}^{2^{s}-1} & b_{s, 5}=x_{1}^{2^{s}-1} x_{3}^{2^{s}-1} & b_{s, 6}=x_{1}^{2^{s}-1} x_{2}^{2^{s}-1}
\end{array}
$$

For $s \geqslant 2$,

$$
\begin{array}{lll}
b_{s, 7}=x_{2} x_{3}^{2^{s}-2} x_{4}^{2^{s}-1} & b_{s, 8}=x_{2} x_{3}^{2^{s}-1} x_{4}^{2^{s}-2} & b_{s, 9}=x_{2}^{2^{s}-1} x_{3} x_{4}^{2^{s}-2} \\
b_{s, 10}=x_{1} x_{3}^{2^{s}-2} x_{4}^{2^{s}-1} & b_{s, 11}=x_{1} x_{3}^{2^{s}-1} x_{4}^{2^{s}-2} & b_{s, 12}=x_{1} x_{2}^{2^{s}-2} x_{4}^{2^{s}-1} \\
b_{s, 13}=x_{1} x_{2}^{2^{s}-2} x_{3}^{2^{s}-1} & b_{s, 14}=x_{1} x_{2}^{2^{s}-1} x_{4}^{2^{s}-2} & b_{s, 15}=x_{1} x_{2}^{2^{s}-1} x_{3}^{2^{s}-2} \\
b_{s, 16}=x_{1}^{2^{s}-1} x_{3} x_{4}^{2^{s}-2} & b_{s, 17}=x_{1}^{2^{s}-1} x_{2} x_{4}^{2^{s}-2} & b_{s, 18}=x_{1}^{2^{s}-1} x_{2} x_{3}^{2^{s}-2}
\end{array}
$$

For $s=2, b_{2,19}=x_{1} x_{2} x_{3}^{2} x_{4}^{2}, b_{2,20}=x_{1} x_{2}^{2} x_{3} x_{4}^{2}$.

For $s \geqslant 3$,

$$
\begin{array}{lll}
b_{s, 19}=x_{2}^{3} x_{3}^{2^{s}-3} x_{4}^{2^{s}-2} & b_{s, 20}=x_{1}^{3} x_{3}^{2^{s}-3} x_{4}^{2^{s}-2} & b_{s, 21}=x_{1}^{3} x_{2}^{2^{s}-3} x_{4}^{2^{s}-2} \\
b_{s, 22}=x_{1}^{3} x_{2}^{2^{s}-3} x_{3}^{2^{s}-2} & b_{s, 23}=x_{1} x_{2}^{2} x_{3}^{s^{s}-4} x_{4}^{2^{s}-1} & b_{s, 24}=x_{1} x_{2}^{2} x_{3}^{2^{s}-1} x_{4}^{2^{s}-4} \\
b_{s, 25}=x_{1} x_{2}^{2^{s}-1} x_{3}^{2} x_{4}^{2^{s}-4} & b_{s, 26}=x_{1}^{2^{2}-1} x_{2} x_{3}^{2} x_{4}^{2^{s}-4} & b_{s, 27}=x_{1} x_{2} x_{3}^{2^{s}-2} x_{4}^{2^{s}-2} \\
b_{s, 28}=x_{1} x_{2}^{2^{s}-2} x_{3} x_{4}^{2^{s}-2} & b_{s, 29}=x_{1}^{3} x_{2}^{5} x_{3}^{2^{s}-6} x_{4}^{2^{s}-4} & b_{s, 30}=x_{1} x_{2}^{2} x_{3}^{2^{s}-3} x_{4}^{2^{s}-2} \\
b_{s, 31}=x_{1} x_{2}^{3} x_{3}^{2^{s}-4} x_{4}^{2^{s}-2} & b_{s, 32}=x_{1} x_{2}^{3} x_{3}^{2^{s}-2} x_{4}^{2^{s}-4} & b_{s, 33}=x_{1}^{3} x_{2} x_{3}^{2^{s}-4} x_{4}^{2^{s}-2} \\
b_{s, 34}=x_{1}^{3} x_{2} x_{3}^{2^{s}-2} x_{4}^{2^{s}-4} & &
\end{array}
$$

For $s=3, b_{3,35}=x_{1}^{3} x_{2}^{3} x_{3}^{4} x_{4}^{4}$, and for $s \geqslant 4, b_{s, 35}=x_{1}^{3} x_{2}^{2^{s}-3} x_{3}^{2} x_{4}^{2^{s}-4}$. 
We set

$$
p_{4, s}= \begin{cases}x_{1} x_{2} x_{3}^{6} x_{4}^{6}+x_{1}^{3} x_{2}^{3} x_{3}^{4} x_{4}^{4}, & \text { if } s=3, \\ \sum_{j=1}^{35} b_{s, j} & \text { if } s \geqslant 4 .\end{cases}
$$

By a direct computation using Proposition 4.2.1, one gets the following.

Proposition 4.2.2. Let $s$ be a positive integer. Then,

$$
\left(\operatorname{Ker}\left(\widetilde{S q}_{*}^{0}\right)_{2^{s+1}-2}\right)^{G L_{4}}= \begin{cases}0, & \text { if } s \leqslant 2, \\ \left\langle\left[p_{4, s}\right]\right\rangle & \text { if } s \geqslant 3 .\end{cases}
$$

For simplicity, we will prove this proposition in detail for $s \geqslant 4$. The others can be proved by the similar computations. We have the following.

Lemma 4.2.3. i) For any $s \geqslant 4$, there is a direct summand decomposition of the $\Sigma_{4}$-modules:

$\left(\operatorname{Ker}\left(\widetilde{S q}_{*}^{0}\right)_{2^{s+1}-2}=\Sigma_{4}\left(b_{s, 1}\right) \oplus \Sigma_{4}\left(b_{s, 7}\right) \oplus \Sigma_{4}\left(b_{s, 19}\right) \oplus \Sigma_{4}\left(b_{s, 23}\right) \oplus \Sigma_{4}\left(b_{s, 29}, b_{s, 30}\right)\right.$.

ii) $\Sigma_{4}\left(b_{s, 1}\right)^{\Sigma_{4}}=\left\langle\left[\bar{p}_{4, s, 1}\right]\right\rangle$, with $\bar{p}_{4, s, 1}=\sum_{j=1}^{6} b_{s, j}$.

iii) $\Sigma_{4}\left(b_{s, 7}\right)^{\Sigma_{4}}=\left\langle\left[\bar{p}_{4, s, 2}\right]\right\rangle$, with $\bar{p}_{4, s, 2}=\sum_{j=7}^{18} b_{s, j}$.

iv) $\Sigma_{4}\left(b_{s, 19}\right)^{\Sigma_{4}}=\left\langle\left[\bar{p}_{4, s, 3}\right]\right\rangle$, with $\bar{p}_{4, s, 3}=\sum_{j=19}^{22} b_{s, j}$.

v) $\Sigma_{4}\left(b_{s, 23}\right)^{\Sigma_{4}}=\left\langle\left[\bar{p}_{4, s, 4}\right]\right\rangle$, with $\bar{p}_{4, s, 4}=\sum_{j=23}^{26} b_{s, j}$.

vi) $\Sigma_{4}\left(b_{s, 29}, a_{s, 30}\right)^{\Sigma_{4}}=\left\langle\left[\bar{p}_{4, s, 5}\right],\left[\bar{p}_{4, s, 6}\right]\right\rangle$, where

$$
\bar{p}_{4, s, 5}=\sum_{j=27}^{29} b_{s, j}, \bar{p}_{4, s, 6}=\sum_{j=30}^{35} b_{s, j} .
$$

Proof. From Proposition 4.2.1 we easily obtain Part i). Now, we prove Part vi) in detail. The others can be proved by the similar computations. By a direct computation we see that the set $\left\{\left[b_{s, j}\right]: j=27 \leqslant j \leqslant 35\right\}$ is a basis for $\Sigma_{4}\left(b_{s, 29}, b_{s, 30}\right)$. Suppose $[f] \in \Sigma_{4}\left(b_{s, 29}, b_{s, 30}\right)^{\Sigma_{4}}$, then $f \equiv \sum_{j=27}^{35} \gamma_{j} b_{s, j}$ with $\gamma_{j} \in \mathbb{F}_{2}$. By a direct computation, we obtain

$$
\begin{aligned}
\rho_{1}(f)+f \equiv & \left(\gamma_{28}+\gamma_{29}+\gamma_{30}+\gamma_{35}\right) b_{s, 27}+\left(\gamma_{31}+\gamma_{33}\right)\left(b_{s, 31}+b_{s, 33}\right) \\
& +\left(\gamma_{32}+\gamma_{34}\right)\left(b_{s, 32}+b_{s, 34}\right) \equiv 0 \\
\rho_{2}(f)+f \equiv & \left(\gamma_{27}+\gamma_{28}+\gamma_{32}+\gamma_{33}\right)\left(b_{s, 27}+b_{s, 28}\right) \\
& +\left(\gamma_{30}+\gamma_{31}\right)\left(b_{s, 30}+b_{s, 31}\right)+\left(\gamma_{34}+\gamma_{35}\right)\left(b_{s, 34}+b_{s, 35}\right) \equiv 0, \\
\rho_{3}(f)+f \equiv & \left(\gamma_{28}+\gamma_{29}+\gamma_{30}+\gamma_{35}\right) b_{s, 27}+\left(\gamma_{31}+\gamma_{32}\right)\left(b_{s, 31}+b_{s, 32}\right) \\
& +\left(\gamma_{33}+\gamma_{34}\right)\left(b_{s, 33}+b_{s, 34}\right) \equiv 0 .
\end{aligned}
$$

The above equalities imply $\gamma_{j}=\gamma_{27}$ for $j=27,28,29$ and $\gamma_{j}=\gamma_{30}$ for $30 \leqslant j \leqslant 35$. The lemma is proved.

Remark 4.2.4. For $s=3$, Parts i) to v) of Lemma 4.2 .3 hold. We replace Part vi) with $\Sigma_{4}\left(b_{3,29}, a_{3,30}\right)^{\Sigma_{4}}=\left\langle\left[p_{4,3}\right]\right\rangle$.

Proof of Proposition 4.2.2. Let $f \in P_{4}$ such that $[f] \in \operatorname{Ker}\left(\widetilde{S q_{*}}\right)_{2^{s+1}-2}^{G L_{4}}$. Then, $[f] \in$ $\operatorname{Ker}\left(\widetilde{S q}_{*}^{0}\right)_{2^{s+1}-2}^{\Sigma_{4}}$. Hence, $f \equiv \sum_{j=1}^{6} \gamma_{j} \bar{p}_{4, s, j}$ with $\gamma_{j} \in \mathbb{F}_{2}$. By a direct computation, 
we have

$$
\begin{aligned}
\rho_{4}(f)+f \equiv & \left(\gamma_{1}+\gamma_{2}\right)\left(b_{s, 2}+b_{s, 3}\right)+\left(\gamma_{2}+\gamma_{4}\right)\left(b_{s, 7}+b_{s, 8}\right)+\left(\gamma_{2}+\gamma_{5}\right) b_{s, 9} \\
& +\left(\gamma_{2}+\gamma_{3}\right)\left(b_{s, 14}+b_{s, 15}\right)+\left(\gamma_{3}+\gamma_{6}\right) b_{s, 19}+\left(\gamma_{4}+\gamma_{6}\right) b_{s, 25} \\
& +\left(\gamma_{2}+\gamma_{3}+\gamma_{4}+\gamma_{5}\right) b_{s, 27}+\left(\gamma_{5}+\gamma_{6}\right)\left(b_{s, 31}+b_{s, 32}\right) \equiv 0 .
\end{aligned}
$$

The last equality implies $\gamma_{j}=\gamma_{1}$ for $1 \leqslant j \leqslant 6$. The proposition follows.

From Theorem 2.1 we have

$$
\operatorname{Ext}_{\mathcal{A}}^{4,2^{s+1}+2}\left(\mathbb{F}_{2}, \mathbb{F}_{2}\right)= \begin{cases}0, & \text { if } s \leqslant 2, \\ \left\langle d_{0}\right\rangle & \text { if } s=3, \\ \left\langle h_{0}^{2} h_{6}^{2}, D_{3}(1)\right\rangle & \text { if } s=6, \\ \left\langle h_{0}^{2} h_{s}^{2}\right\rangle & \text { if } s \geqslant 4, s \neq 6 .\end{cases}
$$

Denote $q_{4, s} \in P\left(\left(P_{4}^{*}\right)_{2^{s+1}-2}\right)$ by setting

$$
\begin{aligned}
q_{4,3}= & a_{1}^{(1)} a_{2}^{(1)} a_{3}^{(6)} a_{4}^{(6)}+a_{1}^{(1)} a_{2}^{(2)} a_{3}^{(5)} a_{4}^{(6)}+a_{1}^{(1)} a_{2}^{(3)} a_{3}^{(4)} a_{4}^{(6)}+a_{1}^{(1)} a_{2}^{(4)} a_{3}^{(3)} a_{4}^{(6)} \\
& +a_{1}^{(1)} a_{2}^{(5)} a_{3}^{(2)} a_{4}^{(6)}+a_{1}^{(1)} a_{2}^{(6)} a_{3}^{(1)} a_{4}^{(6)}+a_{1}^{(2)} a_{2}^{(1)} a_{3}^{(6)} a_{4}^{(5)}+a_{1}^{(2)} a_{2}^{(2)} a_{3}^{(5)} a_{4}^{(5)} \\
& +a_{1}^{(2)} a_{2}^{(3)} a_{3}^{(4)} a_{4}^{(5)}+a_{1}^{(2)} a_{2}^{(4)} a_{3}^{(3)} a_{4}^{(5)}+a_{1}^{(2)} a_{2}^{(5)} a_{3}^{(2)} a_{4}^{(5)}+a_{1}^{(2)} a_{2}^{(6)} a_{3}^{(1)} a_{4}^{(5)} \\
& +a_{1}^{(3)} a_{2}^{(1)} a_{3}^{(5)} a_{4}^{(5)}+a_{1}^{(3)} a_{2}^{(2)} a_{3}^{(6)} a_{4}^{(3)}+a_{1}^{(3)} a_{2}^{(3)} a_{3}^{(2)} a_{4}^{(6)}+a_{1}^{(3)} a_{2}^{(4)} a_{3}^{(1)} a_{4}^{(6)} \\
& +a_{1}^{(3)} a_{2}^{(4)} a_{3}^{(2)} a_{4}^{(5)}+a_{1}^{(3)} a_{2}^{(4)} a_{3}^{(4)} a_{4}^{(3)}+a_{1}^{(3)} a_{2}^{(6)} a_{3}^{(2)} a_{4}^{(3)}+a_{1}^{(4)} a_{2}^{(1)} a_{3}^{(6)} a_{4}^{(3)} \\
& +a_{1}^{(4)} a_{2}^{(2)} a_{3}^{(5)} a_{4}^{(3)}+a_{1}^{(4)} a_{2}^{(3)} a_{3}^{(4)} a_{4}^{(3)}+a_{1}^{(4)} a_{2}^{(4)} a_{3}^{(3)} a_{4}^{(3)}+a_{1}^{(4)} a_{2}^{(5)} a_{3}^{(2)} a_{4}^{(3)} \\
& +a_{1}^{(4)} a_{2}^{(6)} a_{3}^{(1)} a_{4}^{(3)}+a_{1}^{(5)} a_{2}^{(1)} a_{3}^{(3)} a_{4}^{(5)}+a_{1}^{(5)} a_{2}^{(2)} a_{3}^{(1)} a_{4}^{(6)}+a_{1}^{(5)} a_{2}^{(2)} a_{3}^{(2)} a_{4}^{(5)} \\
& +a_{1}^{(5)} a_{2}^{(2)} a_{3}^{(4)} a_{4}^{(3)}+a_{1}^{(5)} a_{2}^{(3)} a_{3}^{(1)} a_{4}^{(5)}+a_{1}^{(5)} a_{2}^{(3)} a_{3}^{(3)} a_{4}^{(3)}+a_{1}^{(5)} a_{2}^{(5)} a_{3}^{(1)} a_{4}^{(3)} \\
& +a_{1}^{(6)} a_{2}^{(1)} a_{3}^{(1)} a_{4}^{(6)}+a_{1}^{(6)} a_{2}^{(1)} a_{3}^{(2)} a_{4}^{(5)}+a_{1}^{(6)} a_{2}^{(1)} a_{3}^{(4)} a_{4}^{(3)}+a_{1}^{(6)} a_{2}^{(2)} a_{3}^{(3)} a_{4}^{(3)},
\end{aligned}
$$

and $q_{4, s}=a_{1}^{(0)} a_{2}^{(0)} a_{3}^{\left(2^{s}-1\right)} a_{4}^{\left(2^{s}-1\right)}$, for $s \geqslant 4$. Since $\left\langle\left[p_{4, s}\right],\left[q_{4, s}\right]\right\rangle=1$ for any $s \geqslant 3$, we get

$$
\mathbb{F}_{2} \otimes_{G L_{4}} P\left(\left(P_{4}\right)_{2^{s+1}-2}^{*}\right)= \begin{cases}0, & \text { if } s \leqslant 2 \\ \left\langle\left[q_{4, s}\right]\right\rangle, & \text { if } s \geqslant 3\end{cases}
$$

By a direct computation, we obtain

$$
\phi_{4}\left(q_{4, s}\right)= \begin{cases}\bar{d}_{0}+\delta\left(\lambda_{1} \lambda_{9} \lambda_{3}^{2}+\lambda_{1} \lambda_{3} \lambda_{9} \lambda_{3}\right), & \text { if } s=3 \\ \lambda_{0}^{2} \lambda_{2}^{2}-1 & \text { if } s>3\end{cases}
$$

From the above equalities and Theorem 2.2, we get

$$
\operatorname{Tr}_{4}\left(\left[q_{4, s}\right]\right)=\left[\phi_{3}\left(q_{4, s}\right)\right]= \begin{cases}{\left[\bar{d}_{0}\right]=d_{0},} & \text { if } s=3, \\ {\left[\lambda_{0}^{2} \lambda_{2^{s}-1}^{2}\right]=h_{0}^{2} h_{s}^{2},} & \text { if } s>3 .\end{cases}
$$

Theorem 4.1 holds for $n=2^{s+1}-2$. 


\subsection{The case $n=2^{s+1}-1$.}

First, we recall the following.

Proposition 4.3.1 (see [20, 23]). Let $n=2^{s+1}-1$ with $s$ a positive integer. Then, the dimension of the $\mathbb{F}_{2}$-vector space $\left(Q P_{4}\right)_{n}$ is determined by the following table:

\begin{tabular}{c|ccccc}
$n=2^{s}-1$ & $s=1$ & $s=2$ & $s=3$ & $s=4$ & $s \geqslant 5$ \\
\hline $\operatorname{dim}\left(Q P_{4}\right)_{n}$ & 14 & 35 & 75 & 89 & 85
\end{tabular}

A basis of $\left(Q P_{4}\right)_{n}$ has been given in [23]. For $s \geqslant k-2$, we set

$$
\eta_{k, s}=\sum_{m=1}^{k-1} \sum_{1 \leqslant i_{1}<\ldots<i_{m} \leqslant k} x_{i_{1}} x_{i_{2}}^{2} \ldots x_{i_{m-1}}^{2^{m-2}} x_{i_{m}}^{2^{s+1}-2^{m-1}} \in\left(P_{k}\right)_{2^{s+1}-1} .
$$

For $k=4$, we denote

$$
\bar{p}_{4, s}= \begin{cases}\eta_{4, s}+x_{1} x_{2}^{2} x_{3}^{2} x_{4}^{2}, & \text { if } s=2, \\ \eta_{4, s}+x_{1} x_{2}^{2} x_{3}^{4} x_{4}^{2^{s+1}-8}, & \text { if } s \geqslant 3 .\end{cases}
$$

By a computation similar to the one in Proposition 4.2.1, one gets the following.

Proposition 4.3.2. Let $s$ be a positive integer. Then,

$$
\left(Q P_{4}\right)_{2^{s+1}-1}^{G L_{4}}= \begin{cases}0, & \text { if } s=1, \\ \left\langle\left[\bar{p}_{4, s}\right]\right\rangle, & \text { if } s \geqslant 2 .\end{cases}
$$

From Theorem 2.1 we have

$$
\operatorname{Ext}_{\mathcal{A}}^{4,2^{s+1}+3}\left(\mathbb{F}_{2}, \mathbb{F}_{2}\right)= \begin{cases}0, & \text { if } s=1, \\ \left\langle h_{0}^{3} h_{s+1}\right\rangle & \text { if } s \geqslant 2 .\end{cases}
$$

Denote $\bar{q}_{4, s}=a_{1}^{(0)} a_{2}^{(0)} a_{2}^{(0)} a_{4}^{\left(2^{s+1}-1\right)} \in P\left(\left(P_{4}^{*}\right)_{2^{s+1}-1}\right)$, for $s \geqslant 2$. It is easy to see that $\left\langle\left[\bar{p}_{4, s}\right],\left[\bar{q}_{4, s}\right]\right\rangle=1$ Hence, we obtain

$$
\mathbb{F}_{2} \otimes_{G L_{4}} P\left(\left(P_{4}\right)_{2^{s+1}-1}^{*}\right)= \begin{cases}0, & \text { if } s=1, \\ \left\langle\left[\bar{q}_{4, s}\right]\right\rangle, & \text { if } s \geqslant 2 .\end{cases}
$$

By a simple computation, we have $\phi_{4}\left(\bar{q}_{4, s}\right)=\lambda_{0}^{3} \lambda_{2^{s+1}-1}$. Hence, using Theorem 2.2, one gets

$$
\operatorname{Tr}_{4}\left(\left[\bar{q}_{4, s}\right]\right)=\left[\phi_{4}\left(\bar{q}_{4, s}\right)\right]=\left[\lambda_{0}^{3} \lambda_{2^{s+1}-1}\right]=h_{0}^{3} h_{s+1} .
$$

Theorem 4.1 is completely proved.

\section{ACKNOWLEDGMENT}

This article was written when the author was visiting the Vietnam Institute for Advanced Study in Mathematics (VIASM) from August to November 2017. He would like to thank the VIASM for supporting the visit, convenient working condition and for kind hospitality.

The author would like to express his warmest thanks to the referee for carefully reading the manuscript and giving many criticisms and suggestions, which have led to an improvement of the article's exposition. 


\section{REFERENCES}

1. J.F. Adams, On the non-existence of elements of Hopf invariant one, Ann. of Math. 72 (1960), 20-104, MR0141119.

2. J. Adem, The iteration of the Steenrod squares in algebraic topology, Proc. Nat. Acad. Sci. U.S.A. 38 (1952), 720-726, MR0050278.

3. J.M. Boardman, Modular representations on the homology of power of real projective space, in: M. C. Tangora (Ed.), Algebraic Topology, Oaxtepec, 1991, in: Contemp. Math., vol. 146, 1993, pp. 49-70, MR1224907.

4. A.K. Bousfield, E.B. Curtis, D.M. Kan, D.G. Quillen, D.L. Rector and J.W. Schlesinger, The mod $p$ lower central series and the Adams spectral sequence, Topology 5 (1966), 331-342, MR0199862 .

5. R.R. Bruner, L.M. Hà and N.H.V. Hưng, On behavior of the algebraic transfer, Trans. Amer. Math. Soc. 357 (2005), 473-487, MR2095619.

6. P.H. Chơn and L.M. Hà, Lambda algebra and the Singer transfer, C. R. Math. Acad. Sci. Paris 349 (2011) 21-23, MR2755689.

7. P.H. Chơn and L.M. Hà, On May spectral sequence and the algebraic transfer, Manuscripta Math. 138 (2012) 141-160, MR2898751.

8. P. H. Chơn and L. M. Hà, On the May spectral sequence and the algebraic transfer II, Topology Appl. 178 (2014) 372-383, MR3276753.

9. L.M. Hà, Sub-Hopf algebras of the Steenrod algebra and the Singer transfer, "Proceedings of the International School and Conference in Algebraic Topology, Hà Nội 2004", Geom. Topol. Monogr., Geom. Topol. Publ., Coventry, vol. 11 (2007), 81-105, MR2402802.

10. N.H.V. Hưng, The weak conjecture on spherical classes, Math. Zeit. 231 (1999) 727-743, MR1709493

11. N.H.V. Hưng, The cohomology of the Steenrod algebra and representations of the general linear groups, Trans. Amer. Math. Soc. 357 (2005), 4065-4089, MR2159700.

12. N.H.V. Hưng and V. T. N. Quỳnh, The image of Singer's fourth transfer, C. R. Math. Acad. Sci. Paris 347 (2009) 1415-1418, MR2588792.

13. M.Kameko, Products of projective spaces as Steenrod modules, PhD. Thesis, The Johns Hopkins University, ProQuest LLC, Ann Arbor, MI, 1990. 29 pp. MR2638633.

14. W.H. Lin, $\operatorname{Ext}_{\mathcal{A}}^{4, *}(\mathbb{Z} / 2, \mathbb{Z} / 2)$ and $\operatorname{Ext}_{\mathcal{A}}^{5, *}(\mathbb{Z} / 2, \mathbb{Z} / 2)$, Topology Appl., 155 (2008) 459-496, MR2380930.

15. N. Minami, The iterated transfer analogue of the new doomsday conjecture, Trans. Amer. Math. Soc. 351 (1999) 2325-2351, MR1443884.

16. T.N. Nam, Transfert algébrique et action du groupe linéaire sur les puissances divisées modulo 2, Ann. Inst. Fourier (Grenoble) 58 (2008) 1785-1837, MR2445834.

17. F.P. Peterson, Generators of $H^{*}\left(\mathbb{R} P^{\infty} \times \mathbb{R} P^{\infty}\right)$ as a module over the Steenrod algebra, Abstracts Amer. Math. Soc. No. 833 (1987) 55-89.

18. W.M. Singer, The transfer in homological algebra, Math. Zeit. 202 (1989) 493-523, MR1022818.

19. N. E. Steenrod and D.B.A. Epstein, Cohomology operations, Annals of Mathematics Studies 50, Princeton University Press, Princeton N.J (1962), MR0145525.

20. N. Sum, The hit problem for the polynomial algebra of four variables, Quy Nhon University, Việt Nam, Preprint 2007, 240 pp., available online at: http://arxiv.org/abs/1412.1709

21. N. Sum, The negative answer to Kameko's conjecture on the hit problem, Adv. Math. 225 (2010), 2365-2390, MR2680169.

22. N. Sum, On the Peterson hit problem of five variables and its applications to the fifth Singer transfer, East-West J. of Mathematics, 16 (2014) 47-62, MR3409252.

23. N. Sum, On the Peterson hit problem, Adv. Math. 274 (2015) 432-489, MR3318156.

24. C.T.C. Wall, Generators and relations for the Steenrod algebra, Annals of Math. 72 (1960), 429-444, MR0116326.

25. J.S.P. Wang On the cohomology of the mod-2 Steenrod algebra and the non-existence of mappings of Hopf invariant one, Illinois Jour. Math. 11 (1967), 480-490, MR0214065.

26. R.M.W. Wood, Steenrod squares of polynomials and the Peterson conjecture, Math. Proc. Cambriges Phil. Soc. 105 (1989), 307-309, MR0974986. 
Department of Mathematics, Quy Nhơn University, 170 An Dương Vương, Quy Nhơn, Bình Định, Viet NAM

Email address: nguyensum@qnu.edu.vn 